\title{
Feature Detection and Curve Fitting Using Fast Walsh Transforms for Shock Tracking: Applications
}

\author{
Peter A Gnoffo* \\ NASA Langley Research Center, Hampton, VA 23681-2199
}

\begin{abstract}
Walsh functions form an orthonormal basis set consisting of square waves. Square waves make the system well suited for detecting and representing functions with discontinuities. Given a uniform distribution of $2^{p}$ cells on a one-dimensional element, it has been proven that the inner product of the Walsh Root function for group $p$ with every polynomial of degree $\leq(p-1)$ across the element is identically zero. It has also been proven that the magnitude and location of a discontinuous jump, as represented by a Heaviside function, are explicitly identified by its Fast Walsh Transform (FWT) coefficients. These two proofs enable an algorithm that quickly provides a Weighted Least Squares fit to distributions across the element that include a discontinuity. The detection of a discontinuity enables analytic relations to locally describe its evolution and provide increased accuracy. Time accurate examples are provided for advection, Burgers equation, and Riemann problems (diaphragm burst) in closed tubes and de Laval nozzles. New algorithms to detect up to two $\mathrm{C} 0$ and/or $\mathrm{C} 1$ discontinuities within a single element are developed for application to the Riemann problem, in which a contact discontinuity and shock wave form after the diaphragm bursts.
\end{abstract}

\section{Introduction}

Accurate simulations of viscous, hypersonic flow generally require high quality grids (hexahedra and prisms) aligned with captured shocks and shear layers. ${ }^{1}$ While there is some evidence that simplex elements (tetrahedra in three-dimensional space) may be acceptable in the context of highorder, discontinuous Galerkin method, ${ }^{2}$ best practice still emphasizes that alignment of cell faces parallel or orthogonal to shocks, contact surfaces, and shear layers are less likely to distort local flow features with adverse effect of computation of gradient-based quantities (heating and shear). Various flavors of multidimensional reconstruction address some limitations of poorly-aligned grids in the vicinity of shocks and contact discontinuities, but these formulations are still not as accurate as well-aligned, hexahedral and/or prismatic grid systems. ${ }^{3,4,5,6}$

Grid alignment becomes more problematic in an unsteady environment. Turbulent boundary layers with hypersonic edge conditions are characterized by shocklets that rapidly form, evolve, and disappear in the vortical structures that transfer mass, momentum, and energy across the layer. ${ }^{7}$ In like manner, supersonic jets interacting with shock-dominated external flow are characterized by rapid, pulsing movement that severely impede the ability to generate prismatic grids that remain aligned to all of the interacting discontinuities. ${ }^{8}$ Best practice in these environments is to align a prismatic grid or fit a moving boundary to the strongest shocks in the domain and then apply well-tuned shock detectors and limiters to weaker shocks that must be captured, regardless of the local quality of grid alignment.

*Senior Research Engineer, Aerothermodynamics Branch; AIAA Fellow 
Is shock capturing with poorly-aligned grids a necessary evil for some, challenging simulations? Localized grid refinement is thought to compensate for associated dissipation errors, but is there a better, more accurate approach? Algorithms to enable detection of discrete features in a simulation, with sufficient accuracy to quantify their evolution across the domain is explored in this paper. In this context, it is not enough to simply detect a feature. Sufficient detail regarding the geometry of the feature must be extracted so that supplementary equations describing feature evolution may be applied. In the case of simple advection, if a discontinuity is detected, it is translated to its new location after time $\Delta t$. In the case of a shock, its pre- and post-shock conditions must be uniquely defined to enable its movement without smearing using a Riemann solver. ${ }^{9}$

In the ideation of an infrastructure for detection and representation of discontinuities, it is logical to examine sets of basis functions that are inherently discontinuous. Walsh functions ${ }^{10,11}$ form such an orthonormal basis set consisting of square waves that are well suited for such feature detection and geometry extraction. ${ }^{12}$ In much the same way that Fast Fourier Transforms (FFT) are used to identify frequency distribution, the Fast Walsh Transform (FWT) can be used to identify features within the distribution. Indeed, the FWT is commonly used in image processing applications. ${ }^{13}$ Recent papers on the use of Walsh functions in the solution of nonlinear partial differential equations (PDEs) have emphasized the ability to derive the solution in transformed space. ${ }^{14,15,16}$ Fundamental operations (multiplication, division, differentiation, and integration) are easily programmed. The multiplication of any two Walsh functions $g_{i}(x)$ and $g_{j}(x)$ with indices $i, j \leq 2^{p}$ is another Walsh function $g_{k}(x)$ with index $k \leq 2^{p}$. This closure property under multiplication enables Walsh function solutions of nonlinear systems of partial differential equations (PDE). However, these Walsh function based solution algorithms showed no significant advantage over the conventional, upwind-based finite-volume techniques they were intended to replace. ${ }^{8}$ The important lesson from these tests is that, while Walsh functions are well suited to representing discontinuous functions, the discontinuities may still be dissipated if the discretization algorithm is not formulated in a way that preserves the detected features.

The vision for the FWT fit is to work like a shock-capturing method with respect to detection but work like a shock-fitting method with respect to use of more accurate analytic relations. The long term goal of this work is to enable accurate simulations of viscous, hypersonic flow on simplex elements (triangles in 2D, tetrahedra in 3D). For steady problems, the objective is accuracy that meets or exceeds that obtained on a well-aligned prismatic grid system. For unsteady problems, the objective is to create an infrastructure that accurately detects rapidly evolving shocklets and computes their movement across the domain using analytic jump conditions. The goal requires: (1) the ability to compute viscous quantities (heating, shear) on simplex elements that are not generally well-aligned with the shear layer, and (2) the ability to detect shocks and maintain proper jump conditions on poorly-aligned grids. Much progress has been realized toward the first goal through a hyberbolic formulation of the governing equations that enables highly accurate resolution of gradients on simplex elements. ${ }^{17}$ Accuracy has been demonstrated on such grids with randomized perturbations that intentionally disrupt grid quality. ${ }^{18}$ This paper is the second of two to address the detection and tracking of discontinuities with Walsh functions. The first paper $^{19}$ developed the mathematical foundation for using Fast Walsh Transforms (FWT) to detect discontinuities while simultaneously providing a baseline polynomial fit to smooth regions spanning a computational element. The most fundamental aspects of feature detection are developed there for one-dimensional simulations. This paper reviews highlights of the first and then presents new material on the time accurate detection and tracking of discontinuities for the Euler equations. It essentially seeks to replace shock capturing with shock fitting. ${ }^{20,21}$ Preliminary investigation suggests that multidimensional extensions could utilize a multidimensional Walsh function series using the same fractal recursion formulation on simplex elements that is already defined for the one-dimensional series. 
This paper is organized as follows. Walsh function fundamentals are reviewed in II. Highlights from the first paper ${ }^{19}$ describing feature detection and algorithm formulation using Walsh functions are reviewed in Sec. III. The theorem addressing Walsh Root Function annihilation of low-order polynomials is discussed here. It becomes the cornerstone for a Weighted Least Squares Fit based on the FWT, with results presented in Sec. IV. Feature detection in the presence of several offnominal conditions, such as noisy distributions or two discontinuities, is discussed in Sec. V. The principal focus of this paper is to enable preservation and tracking of discontinuities that develop in the solution of a time-accurate PDE by exploiting the FWT capabilities for feature detection. To this end, a conservative formulation of one-dimensional equations are presented in Sec. VI. The key innovation here is to formulate the flux relative to the FWT curve fit, including the discontinuity. All corrections to the baseline fit are then based on a $C_{0}$ continuous baseline. The new algorithm is tested through simulations of time-accurate advection, Burgers' equation, and quasi-one-dimensional nozzle flow and shock tube flow in Sec. VII. Concluding remarks and directions for future research are finally presented in Sec. VIII.

\section{Walsh Function Fundamentals}

Let $g_{n}(x)$ be a basis function over the domain $x_{a} \leq x \leq x_{b}$ with $n$ segments. The orthonormal basis function requirements ${ }^{22}$ that

$$
\int_{x_{a}}^{x_{b}} g_{n}(x) g_{m}(x) d x=\delta_{n m}
$$

enable a recursive algorithm to define $g_{n}(x)$ based on the following constraints. Constrain $\left|g_{n}(x)\right|$ equal to a constant across the entire domain (i.e., only allow square waves of constant amplitude). Let index $p \geq 0$ define a segment size $d x_{p}$.

$$
d x_{p}=\left(x_{b}-x_{a}\right) / 2^{p}
$$

An additional constraint on segment lengths within $g_{n}(x)$ requires that at most two segment lengths $\left(d x_{p}\right.$ and $\left.2 d x_{p}\right)$ are allowed to create $n$ segments spanning the domain. These new constraints allow basis functions to be grouped according to the smallest segment size. A basis function $g_{n}(x)$ belongs to group $p$ if

$$
2^{p-1}<n \leq 2^{p}
$$

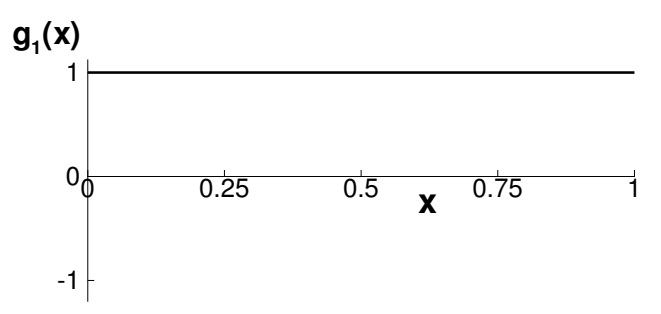

Figure 1. Basis function in group $p=0$.

As an example, consider the evolution of the first four groups of $g_{n}(x)$ corresponding to $0 \leq p \leq 3$ on the domain with $x_{a}=0$ and $x_{b}=1$. For $p=0$, the only element of the group according to Eq. 3 is $n=1$. A single segment with length given by Eq. 2 spans the domain. Therefore, $g_{1}(x)=1$ (Fig. 1 ) is the first basis function, belonging to group $p=0$ and satisfying Eq. 1. (While $g_{1}(x)=-1$ 


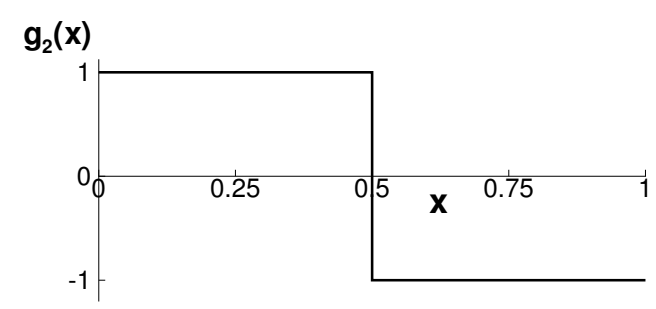

Figure 2. Basis function in group $p=1$.

also satisfies Eq. 1, it is convenient to adopt a convention where the sign of the function on the first segment is positive.)

For $p=1$, the only element of the group according to Eq. 3 is $n=2$. Two segments with length equal to $1 / 2$ span the domain. Therefore,

$$
g_{2}(x)=\left\{\begin{array}{rll}
1 & \text { for } & 0 \leq x<\frac{1}{2} \\
-1 & \text { for } & \frac{1}{2} \leq x<1
\end{array}\right.
$$

where function $g_{2}(x)$ (Fig. 2) is the second basis function, belonging to group $p=1$ and satisfying Eq. 1. The value of the function at interior segment boundaries is set to 0 , the average of the function on either side of the boundary. (The vertical line through interior segment boundaries in Fig. 2 provides strong visual impact of the discontinuity and is not meant to imply all values between -1 and 1 are included.)

The group defined by $p=2$ is the first to have more than one function, $g_{3}(x)$ and $g_{4}(x)$. The allowed segment sizes in this group are $1 / 4$ and $1 / 2$. Each function is initialized with the smallest segment at the beginning and end of the domain. For $n=3$, this initialization leaves a single segment of length $1 / 2$ available to span the interior of the domain. For $n=4$, the initialization leaves two segments of length $1 / 4$ to span the interior of the domain. The corresponding functions are shown in Fig. 3. By inspection, it is clear that these first four basis functions satisfy Eq. 1.

$$
g_{3}(x)=\left\{\begin{array}{rll}
1 & \text { for } & 0 \leq x<\frac{1}{4} \\
-1 & \text { for } & \frac{1}{4} \leq x<\frac{3}{4} \\
1 & \text { for } & \frac{3}{4} \leq x<1
\end{array} \quad g_{4}(x)=\left\{\begin{array}{rcc}
1 & \text { for } & 0 \leq x<\frac{1}{4} \\
-1 & \text { for } & \frac{1}{4} \leq x<\frac{1}{2} \\
1 & \text { for } & \frac{1}{2} \leq x<\frac{3}{4} \\
-1 & \text { for } & \frac{3}{4} \leq x<1
\end{array}\right.\right.
$$

The group defined by $p=3$ includes four functions, $g_{5}(x)$ through $g_{8}(x)$. The allowed segment sizes in this group are $1 / 8$ and $1 / 4$. Each function is again initialized with the smallest segment at the beginning and end of the domain. This is the first group where the distribution of segments required to satisfy Eq. 1 for all combinations of functions in groups $0-3$ becomes nontrivial. Still, the number of permutations of segment size distribution is small and a solution, evident by inspection, is shown in Fig. 4.

A fractal-based recursion algorithm is used to define the wave pattern for ever larger numbers of segments. ${ }^{16}$ A function $f(x)$ may now be represented by an infinite series

$$
\sum_{n=1}^{\infty} A_{n} g_{n}(x)
$$




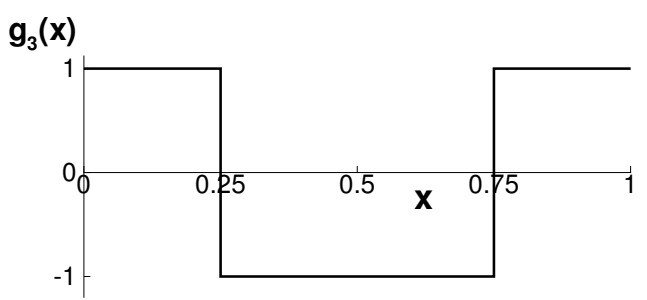

(a) $g_{3}(x)$

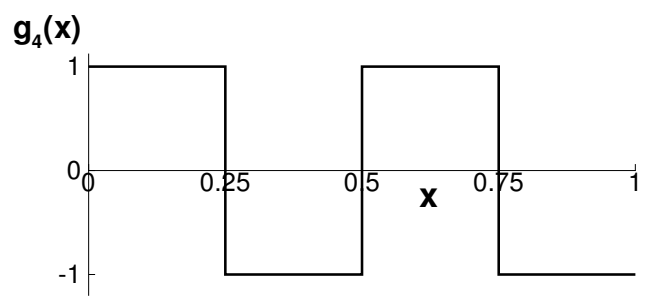

(b) $g_{4}(x)$

Figure 3. Basis functions in group $p=2$.

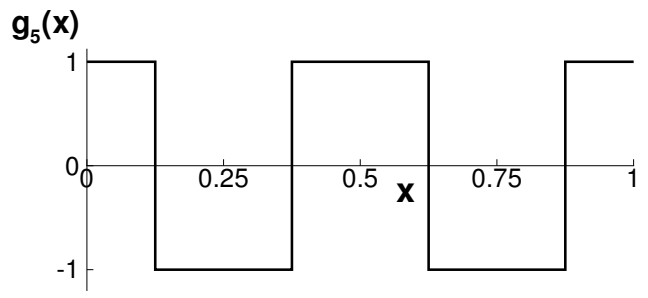

(a) $g_{5}(x)$

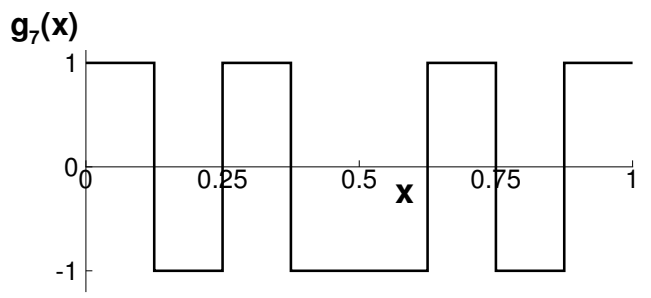

(c) $g_{7}(x)$

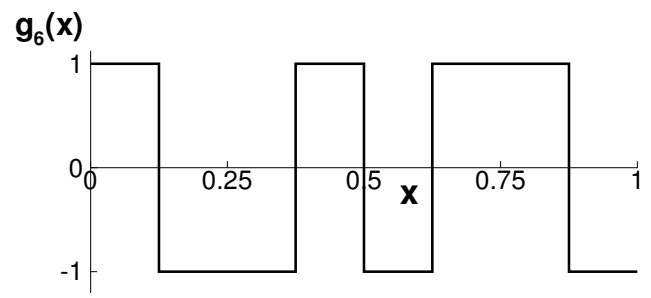

(b) $g_{6}(x)$

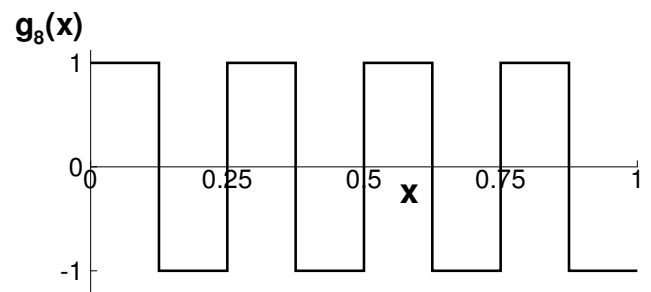

(d) $g_{8}(x)$

Figure 4. Basis functions in group $p=3$.

over the interval $x_{a} \leq x \leq x_{b}$. The coefficients $A_{n}$ are computed

$$
A_{n}=\int_{x_{a}}^{x_{b}} f(x) g_{n}(x) d x
$$

Note that, for domains comprised of a maximum $N=2^{p}$ segments, the Fast Walsh Transform $(\mathrm{FWT})^{19}$ will be used to compute the wave components $A_{n}$ from $f_{n}$ in order $p N$ operations. If $f_{n}=$

$\int_{x_{n}-d x / 2}^{x_{n}+d x / 2} f(x) d x$ (integrated average), then a continuous representation is defined. If $f_{n}=f\left(x_{n}\right)$, then a discrete representation is defined. In the derivations that follow, a discrete representation is assumed.

\section{Feature Detection from the Fractal Fingerprint}

The fractal nature of the Walsh basis functions ${ }^{16}$ introduces repeated patterns in the wave component magnitudes that can be exploited for many applications. These patterns are accentuated in a mapping defined as the fractal fingerprint (FFP). The FFP has been presented in detail 
previously $^{19}$ and highlights from that work are summarized here.

- Walsh functions in group $p$ are simply step functions with a baseline step size equal to $\left(x_{b}-x_{a}\right) / 2^{p}$ and a maximum of two adjacent steps with the same sign. A mapping that retains group identity for the plot of $A_{n}$ in the FFP is defined:

$$
m=2^{p}+1-n \quad \text { for } \quad 2^{p-1}<n \leq 2^{p}
$$

- For singularity-free functions, a recursive algorithm is evident that enables prolongation - an estimation of all but one element, $r(p)$, of a group $p$ coefficient from a given group $p-1$. The new, unique element $r$ is defined as the root index and the Walsh function with root index $r$ is defined as the Walsh root function $g_{r(p)}(x)$.

- It is proven ${ }^{19}$ that the inner product of the Walsh root function $g_{r(p)}(x)$ with any polynomial of degree $p-1$ over the interval $x_{a} \leq x \leq x_{b}$ is identically zero. This proof enables an efficient algorithm for a least squares polynomial fit through data sets spanning $2^{p}$ cells in the interval.

- Coefficients of the polynomial curve fit,

$$
\tilde{f}(x)=\sum_{k=0}^{m} a_{k} x^{k}
$$

to the distribution $f\left(x_{i}\right)$ for $0<i \leq 2^{p}$ are computed from

$$
\left[\begin{array}{ccccc}
B_{r(0), 0} & B_{r(0), 1} & B_{r(0), 2} & \cdots & B_{r(0), m} \\
0 & B_{r(1), 1} & B_{r(1), 2} & \cdots & B_{r(1), m} \\
0 & 0 & B_{r(2), 2} & \cdots & B_{r(2), m} \\
\vdots & \vdots & \vdots & \ddots & \vdots \\
0 & 0 & 0 & \cdots & B_{r(m), m}
\end{array}\right]\left[\begin{array}{c}
a_{0} \\
a_{1} \\
a_{2} \\
\vdots \\
a_{m}
\end{array}\right]=\left[\begin{array}{c}
A_{r(0)} \\
A_{r(1)} \\
A_{r(2)} \\
\vdots \\
A_{r(m)}
\end{array}\right]
$$

where $A_{r(m)}$ are the coefficients of the Walsh root functions obtained through the Fast Walsh Transform (FWT) of $f\left(x_{i}\right)$ and $B_{r(n), m}$ are constants, equal to the coefficients of the Walsh root functions obtained through the FWT of $x_{i}^{n}$.

- Given a distribution, $f\left(x_{i}\right)$, and a shifted distribution $f\left(x_{i}+\Delta x\right)$, across $2^{p}$ cells on an interval $x_{a} \leq x \leq x_{b}$, two essential properties of their FFPs can be identified to enable feature detection of a discontinuity with a jump magnitude greater than $|h|$.

- The average of $\left|A_{n}\right|$ for $2^{p-1}<n \leq 2^{p}$ must be greater than $|h| \sqrt{x_{b}-x_{a}} / 2^{p}$

- The pattern of sign changes of $A_{n}$ for $2^{p-1}<n \leq 2^{p}$ determines the location of the discontinuity within the interval

- A distribution with a $C_{0}$ discontinuity may be approximated by

$$
\tilde{f}(x)=\sum_{k=0}^{m} a_{k} x^{k}+h H\left(x, x^{*}\right)
$$

where

$$
H\left(x, x^{*}\right) \equiv\left\{\begin{array}{lll}
0 & \text { for } & x \leq x^{*} \\
1 & \text { for } & x>x^{*}
\end{array}\right.
$$

The coefficients $a_{k}$ are determined by subtracting the influence of a detected shock from a baseline shocked distribution. 
- First, compute and store the constant Walsh Root function coefficients $B_{r(\kappa), k}^{*}$ for $H\left(x_{i}, x^{*}\right)$ for every ordered pair $\left(x_{k}, x_{k+1}\right)$ bounding a shock location $x^{*}$ occurring in the interval $\left(x_{a}, x_{b}\right)$.

$$
B_{r(\kappa), k}^{*}=\sum_{i=1}^{2^{p}} g_{r(\kappa)}\left(x_{i}\right) H\left(x_{i}, x^{*}\right) \Delta x \quad x_{a} \leq x_{k}<x^{*}<x_{k+1} \leq x_{b} \quad 0 \leq \kappa \leq p
$$

- Second, the Walsh Root coefficients for a smooth distribution $A_{r(\kappa)}$ are now computed in Eq. 12 from the Walsh Root coefficients of the shocked distribution $A_{r(\kappa)}^{*}$ minus the rescaled Walsh Root Function coefficients for the Heaviside function $B_{r(\kappa), k}^{*}$ with the shock location given by index $k$.

$$
A_{r(\kappa)}=A_{r(\kappa)}^{*}-h B_{r(\kappa), k}^{*}
$$

\section{Polynomial Curve Fits from the Walsh Transform}

Representative curve fits for several functions are presented in Fig. 5 . The discrete data $f_{i}$ being fit are shown as black squares. Curve fits through the data are shown as a black dashed line for the 4th degree polynomial $(m=4)$ and as black solid line for the 6th degree polynomial $(m=6)$. Both lines are contained within the symbols for each example. The difference between the discrete data and the curve fits are shown on the right scale with red lines. The difference is identically zero for $f_{i}=x_{i}^{4}$ in Fig. 5a because the algorithm in Eqs. 8 will exactly recover the polynomial coefficients if $f_{i}$ is formed from a polynomial of degree less than or equal to $m$, the degree of $\tilde{f}\left(x_{i}\right)$. Errors associated with lack of precision begin to manifest at $m>8$ - the Walsh Root Function coefficient associated with $r(9)=342$ tends to have a very small magnitude (related to the sum $2^{8}$ differences of nearly equal quantities) and this loss of precision directly affects the calculation of the leading coefficient $a_{9}$.

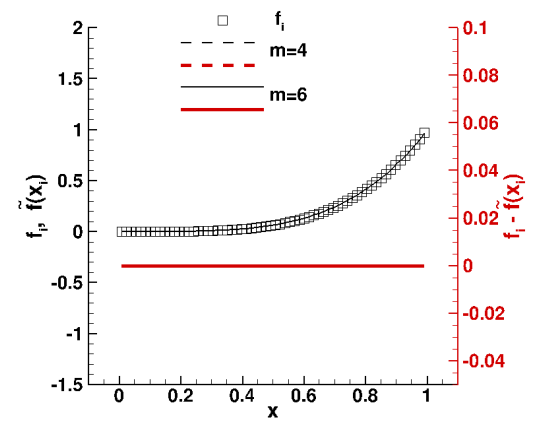

(a) $f_{i}=x_{i}^{4}$

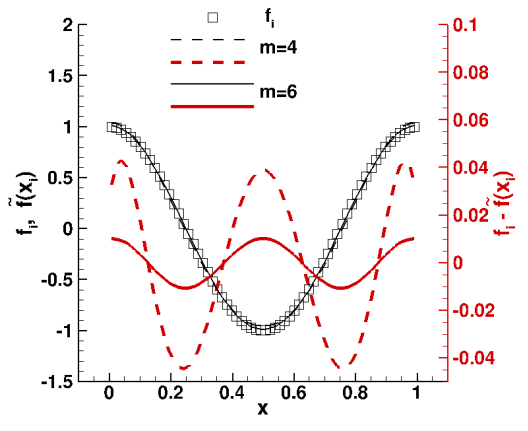

(b) $f_{i}=\cos \left(2 \pi x_{i}\right)$

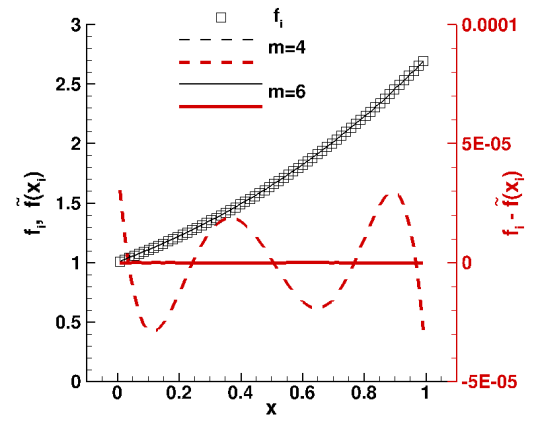

(c) $f_{i}=\exp \left(x_{i}\right)$

Figure 5. Comparison of polynomial curve fit $\tilde{f}\left(x_{i}\right)=\sum_{k=0}^{m} a_{k} x_{i}^{k}$ (black lines) to singularity-free functions, $f_{i}$ (square symbols) with $1 \leq i \leq 64(p=6)$. Their difference is shown on the right scale for polynomials of degree 4 (dashed red line) and degree 6 (solid red line).

The value of $f_{i+1 / 2}$ at the interface of two cells will be required in subsequent simulations in Sec. VI. To assess accuracy of interface interpolation, formulations of the interface value are compared to the special case where an exact interface value $f_{e, i+1 / 2}$ is available. Cell interface values will be computed on the basis of the fit value at the interface, $\tilde{f}\left(x_{i+1 / 2}\right)$, and interpolated corrections from neighbor cells, $\left(f_{i}-\tilde{f}\left(x_{i}\right)\right)$. Consequently, the interface value is a function of the 
curve fit polynomial $\tilde{f}(x)$ of degree $m$ that is fit to $2^{p}$ discrete points where $m \leq p$. A third-order interpolation with this property is defined below in Eq. 13.

$$
\begin{aligned}
& f_{i+1 / 2}=\tilde{f}\left(x_{i+1 / 2}\right)+\frac{1}{8}\left[3\left(f_{i+1}-\tilde{f}\left(x_{i+1}\right)\right)+6\left(f_{i}-\tilde{f}\left(x_{i}\right)\right)-\left(f_{i-2}-\tilde{f}\left(x_{i-2}\right)\right)\right] \\
& =\frac{1}{8}\left(3 f_{i+1}+6 f_{i}-f_{i-1}\right)-\frac{1}{2}\left(\tilde{f}\left(x_{i+1}\right)-2 \tilde{f}\left(x_{i+1 / 2}\right)+\tilde{f}\left(x_{i}\right)\right) \\
& +\frac{1}{8}\left(\tilde{f}\left(x_{i+1}\right)-2 \tilde{f}\left(x_{i}\right)+\tilde{f}\left(x_{i-1}\right)\right) \quad \text { 3rd order }
\end{aligned}
$$

The error norms $\left(L_{1}\right)$ of the corrected interface values from Eq. 13 are defined in Eq. 14. The error norms of a reference, uncorrected interpolation formulation $\left(L_{1, \text { ref }}\right)$ are defined in Eq. 15.

$$
\begin{gathered}
L_{1}=\sum_{i=1}^{2^{p}-1}\left|f_{i+1 / 2}-f_{e, i+1 / 2}\right| \Delta x \\
L_{1, \text { ref }}=\sum_{i=1}^{2^{p}-1}\left|\frac{1}{8}\left(3 f_{i+1}+6 f_{i}-f_{i-1}\right)-f_{e, i+1 / 2}\right| \Delta x
\end{gathered}
$$

Note that $x_{i+1 / 2}=\left(x_{i}+x_{i+1}\right) / 2$ and $f_{e, i+1 / 2}$ is the interface value for one of three test functions $\left(\exp \left(x_{i+1 / 2}\right), \cos \left(2 \pi x_{i+1 / 2}\right)\right.$ or $\left.1 /\left(1+x_{i+1 / 2}\right)\right)$. The last function here replaces the monomial $x_{i}^{4}$ because the Walsh transform fit identically reproduces this function.

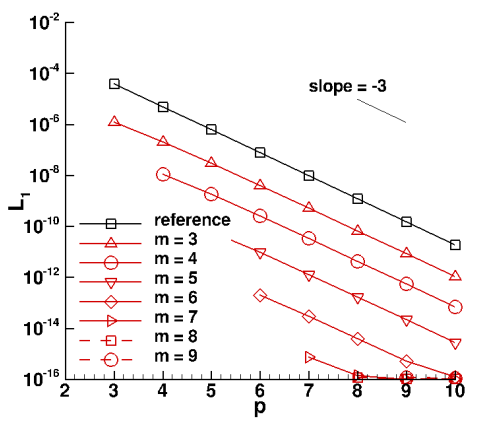

(a) $f_{i}=\exp \left(x_{i}\right)$

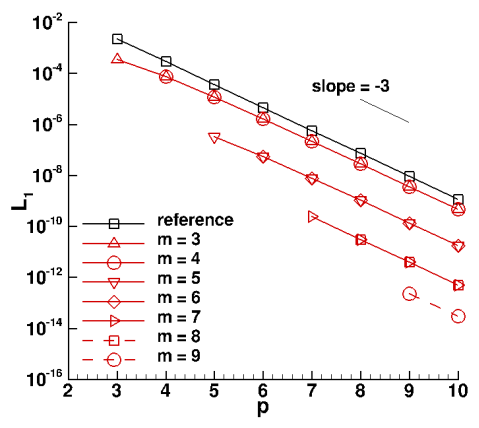

(b) $f_{i}=\cos \left(2 \pi x_{i}\right)$

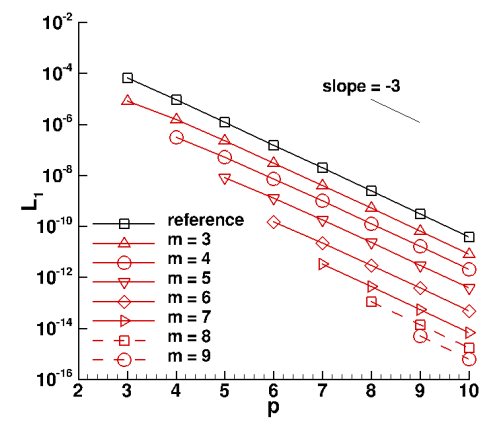

(c) $f_{i}=1 /\left(1+x_{i}\right)$

Figure 6. Variation of $L_{1}$ error norm with $p$ for 3rd-order accurate interface interpolation on the interval $0 \leq x \leq 0.5$ with $2^{p}$ discrete points comparing conventional interpolation (black line) to Walsh Transform curve fit corrected interpolation (red lines) for polynomial fits varying from degree 3 to degree $p(3 \leq m \leq p)$.

It is instructive to compare the error norms of the corrected interpolation formulations from Eq. 14 to the error norms of the reference, uncorrected interpolation formulation in Eq. 15 in Fig. 6. In these figures, each increment in $p$ corresponds to a factor of two decrease in the mesh size. A slope of -3 in Fig. 6 indicates that the error norm drops by a factor of eight for an increment in $p$ equal to one. All of the interpolation formulations are third-order accurate except for the $m>6$ in Fig. 6a in which the formulation bumps up against machine zero capability with double precision arithmetic. Orders of magnitude increase in accuracy are demonstrated as the order of the polynomial fit $m$ increases for the correction terms. Note that in Fig. $6 \mathrm{~b}$ the variation of error norms is nearly identical for $m=3$ or $4, m=5$ or 6 , and $m=7$ or 8 . This behavior derives from 
the fact that the even powers of $x$ in the fit to $\cos (2 \pi x)$ are almost identically zero on the interval $0 \leq x \leq 0.5$. Consequently, the next even power of the curve fit provides no new information beyond the previous odd power.

The rate of improvement with increasing $m$ depends on the quality of the curve fit. The red lines are generally observed to lie below the reference black lines for smooth, monotone functions. Even if the function has a local maximum or minimum, improvement is usually noted unless an attempt is made to capture distributions with multiple extrema.

Finally, the operation count required to compute the correction terms is a relatively small overhead with respect to that required for the reference interface interpolation. Evaluation of the polynomial coefficients in Eq. 8 requires order $p 2^{p}$ operations; however, this single fit is used to compute the correction to $2^{p}-1$ interface values.

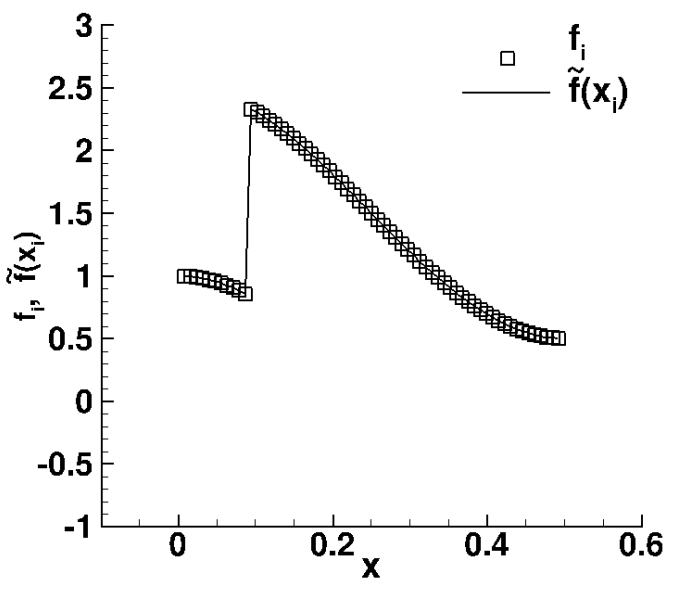

(a) original distribution and curve fit, $h=1.5$

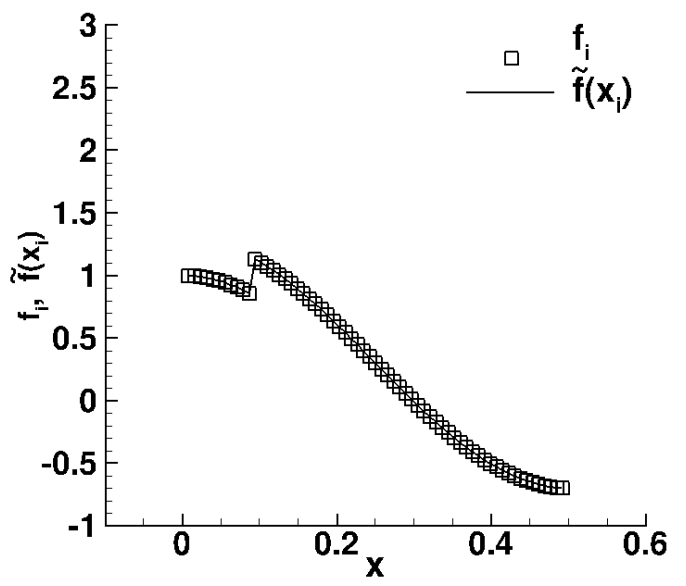

(c) original distribution and curve fit, $h=0.3$

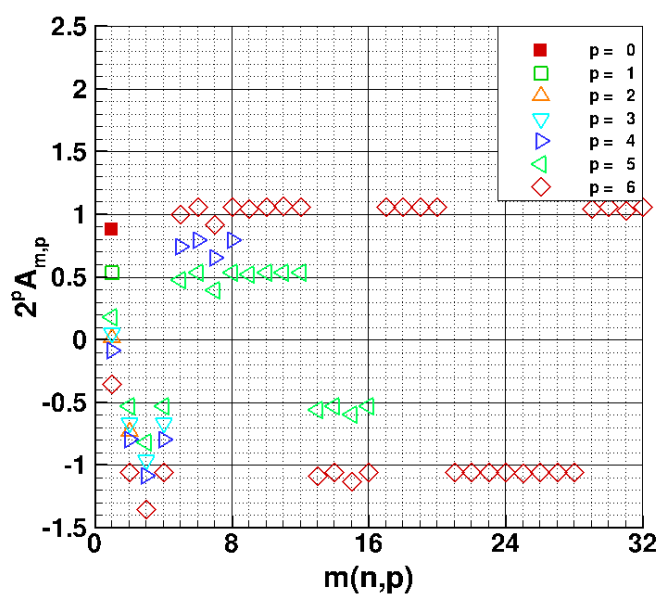

(b) FFP, $h=1.5$

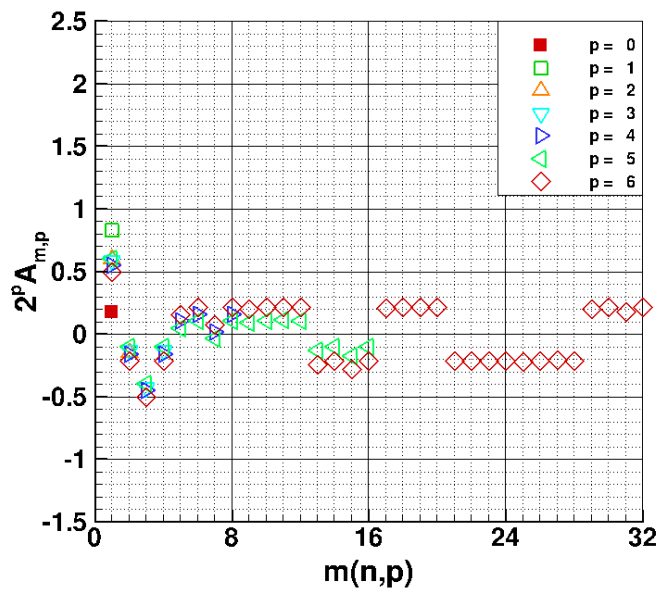

(d) FFP, $h=0.3$

Figure 7. Feature detection within the distribution given by Eq. 16 with discontinuity at $x_{11}<x^{*}<x_{12}$.

The problem of detecting a shock in the context of a more general, underlying distribution is 
exemplified in Fig. 7 with distribution defined in Eq. 16.

$$
f_{i}=\cos \left(2 \pi x_{i}\right)+h H\left(x_{i}, x^{*}\right) \quad \text { for } \quad 0 \leq x_{i} \leq 1, \quad x_{i}=\left(i-\frac{1}{2}\right) \Delta x, \quad \Delta x=\frac{1}{64}
$$

Examples of curve fits $\tilde{f}\left(x_{i}\right)$ including the detected shock location are provided with solid black lines in Figs. $7 \mathrm{a}$ and $7 \mathrm{c}$. The corresponding FFPs used to derive these fits are shown in Figs. $7 \mathrm{~b}$ and $7 \mathrm{~d}$.

\section{Special Cases}

\section{A. Noisy Distributions}

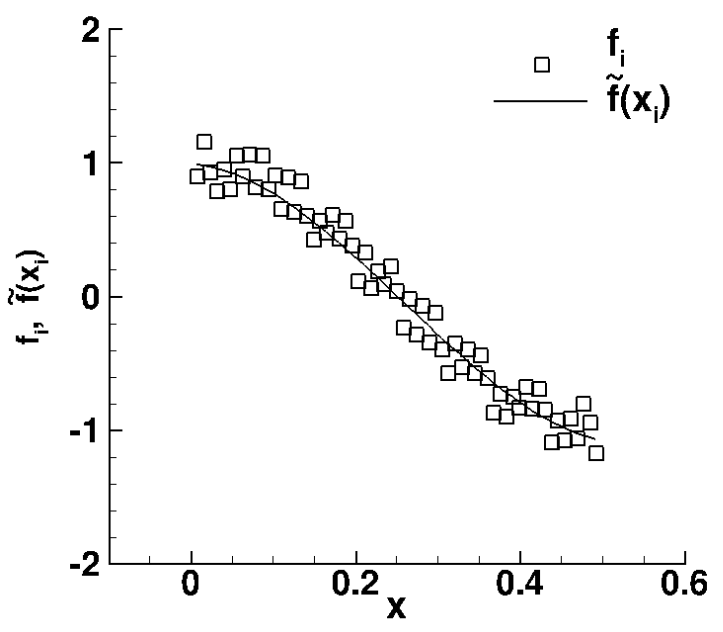

(a) noisy distribution and curve fit with $m=3$

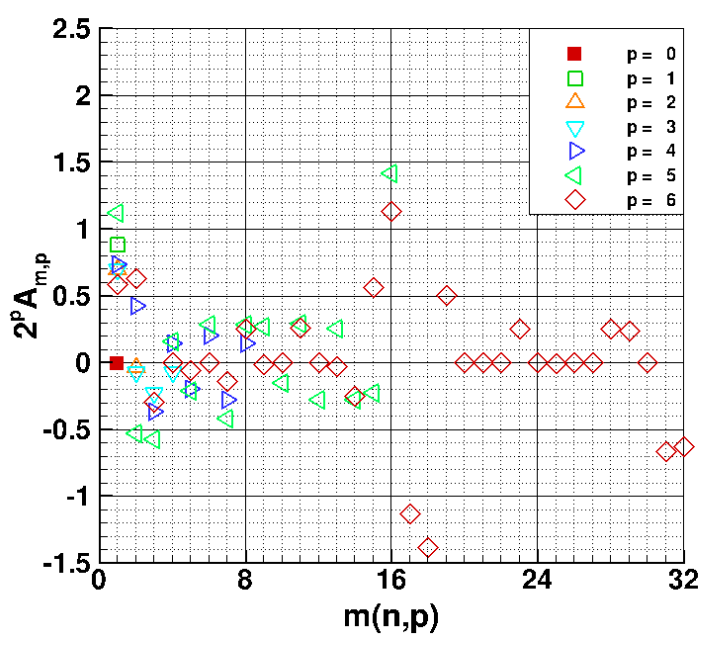

(b) FFP of noisy distribution

Figure 8. Curve fit and FFP of distribution given by $f_{i}=\cos \left(2 \pi x_{i}\right)+0.2\left(\operatorname{rnd}_{i}-0.5\right) 2$ on interval $(\mathbf{0 , 0 . 5})$.

Random numbers with magnitude $0 \leq \operatorname{rnd}_{i} \leq 1$ are mapped to the function $f_{i}=\cos \left(2 \pi x_{i}\right)$ on the interval $(0,0.5)$ in order to test the algorithms for determining polynomial fits and feature detection on noisy distributions. If $m \geq p-1$ in Eq. 7 and the distribution $f_{i}$ is noisy, then it is usually observed that the resulting curve fit using the algorithm defined by Eq. 8 is poor. The magnitude of the highest-order polynomial coefficient $a_{m}$ in such a case is usually too large and the fit oscillates through the distribution with large excursions above and below the mean. It is found that $m \leq 4$ through distributions with $p \geq 6$ produce good curve fits through noisy data, as shown in Fig. 8. The noise is effectively filtered by basing the curve fit on the truncated Walsh function transform.

The ability to detect a shock in the presence of a noisy distribution is demonstrated in Fig. 9. The two randomly generated test cases presented in Fig. 9 highlight the challenge of detecting a shock in the presence of noise with amplitude equal to 0.2 . In most cases, a result consistent with Random Sample 1 in Fig. 9a is obtained. The pattern match in the FFP (Fig. 9b) is somewhat disrupted by noise; however, the disruption was not sufficient to thwart the detection algorithm. Occasionally, the randomly generated data produces an FFP with too many disruptions to an accepted pattern to identify a shock location, as shown in Fig. 9c and d. A similar test in 


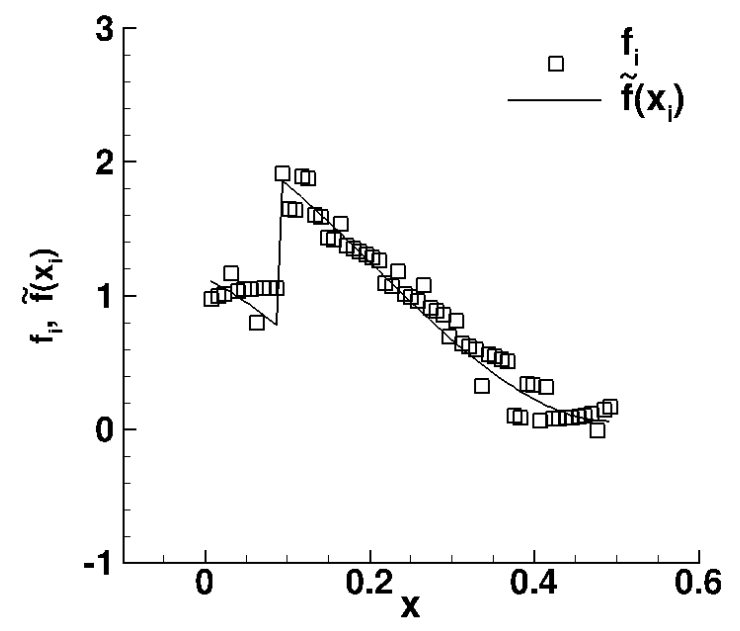

(a) Random sample 1: noisy distribution with shock jump $h=1.0$ and curve fit with $m=3$

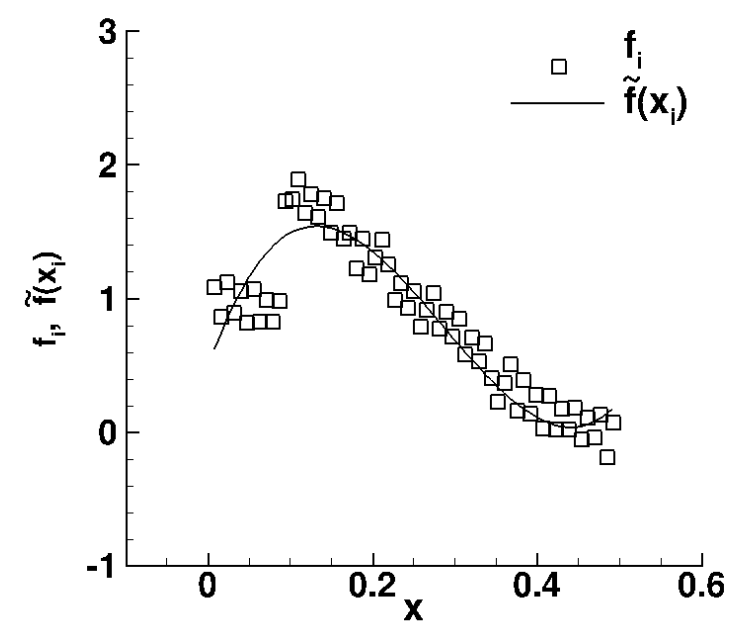

(c) Random Sample 2: noisy distribution with shock jump $h=1.0$ and curve fit with $m=3$

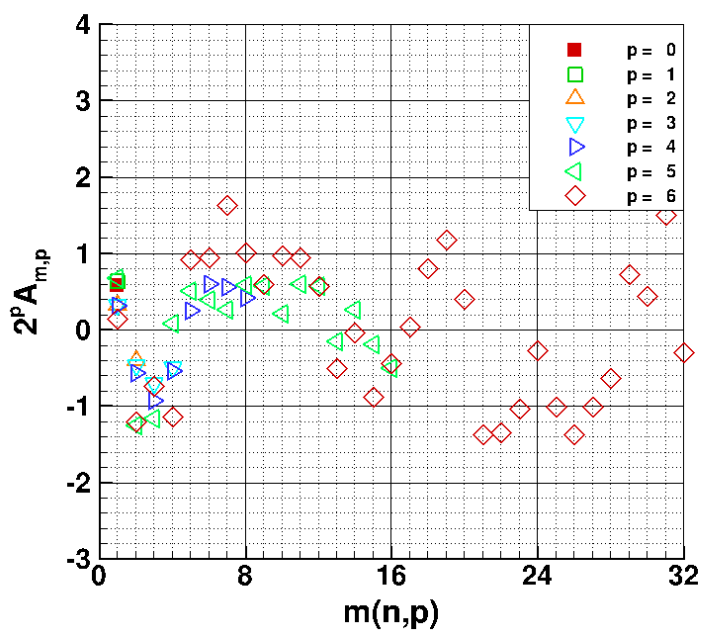

(b) Random sample 1: FFP of noisy distribution with shock jump $h=1.0$

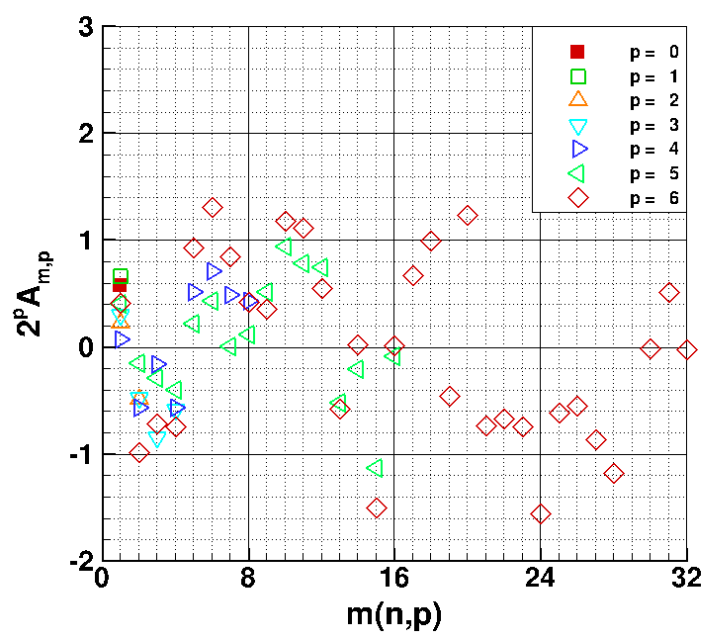

(d) Random Sample 2: FFP of noisy distribution with shock jump $h=1.0$

Figure 9. Curve fit and FFP of distribution given by $f_{i}=\cos \left(2 \pi x_{i}\right)+H\left(x_{i}, x^{*}\right)+0.2\left(\mathbf{r n d}_{i}-0.5\right) 2$ on interval $(0,0.5)$ with $x_{11}<x^{*}<x_{12}$.

which the amplitude of the noise was reduced to 0.1 encountered no failures of the shock detection algorithm.

\section{B. Distributions with Two C0 or C1 Discontinuities}

Feature detection in a hypersonic flow solver must be able to accommodate the interaction of two shocks in the same element. An analysis of the FFP for two Heaviside functions reveals that the resulting distribution of Walsh coefficients is nothing more than a linear combination of each individual function's coefficients. A bilevel distribution of the group $p$ coefficients is observed, from 
which it is possible to reconstruct the effect of each Heaviside function individually. A representative curve fit is shown in Fig. 10a.

In like manner, the detection of $\mathrm{C} 1$ discontinuities is accommodated by taking the derivative of the transform ${ }^{16}$ and then search as before for the $\mathrm{C} 0$ discontinuities. The differentiation operator is simply a matrix multiply of the Walsh coefficients. An example with $\mathrm{C} 1$ (slope) discontinuities is generated with $H^{\prime}\left(x, x^{*}\right)=\left(x-x^{*}\right) H\left(x, x^{*}\right)$ and is presented in Fig. 10b.

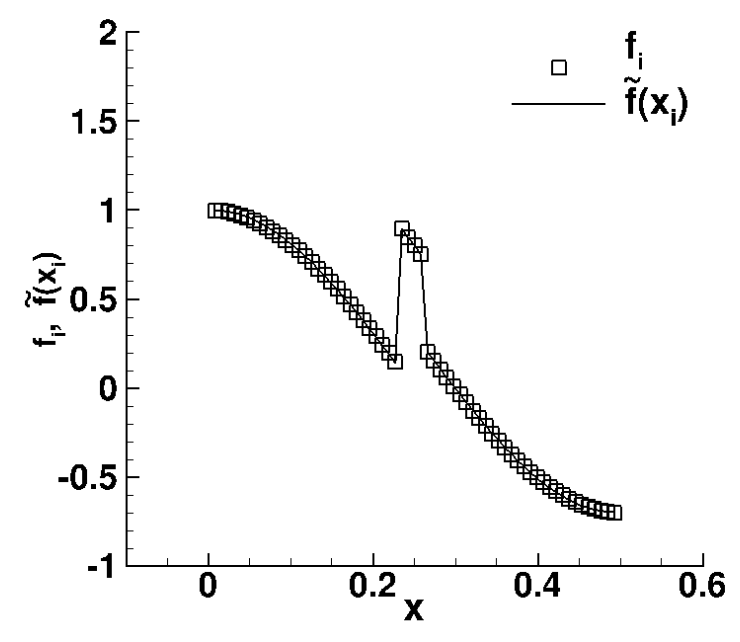

(a) $f_{i}=\cos \left(2 \pi x_{i}\right)+0.8 H\left(x, x_{1}^{*}\right)-0.5 H\left(x, x_{2}^{*}\right)$

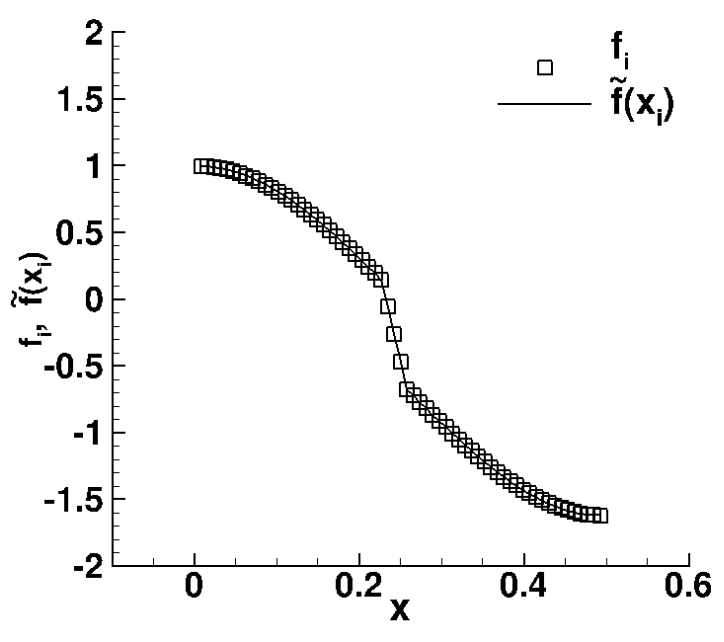

(b) $f_{i}=\cos \left(2 \pi x_{i}\right)-20 H^{\prime}\left(x, x_{1}^{*}\right)+20 H^{\prime}\left(x, x_{2}^{*}\right)$

Figure 10. An example of feature detection for a two discontinuity distribution with $x_{29}<x_{1}^{*}<x_{30}$ and $x_{33}<x_{2}^{*}<x_{34}$ for $p=6$ and $m=4$.

\section{Formulation Relative to the FWT Fit}

The FWT curve fit with feature detection provides new opportunities for the solution of differential equations. Simple examples are considered in this section. The essence of the approach is to linearize the system of equations with respect to the FWT fit.

The value of any variable calculated from the reference FWT fit is indicated with a tilde over the variable name. The formulation of any interface value at index $i+1 / 2$ between indices $i$ and $i+1$ requires a single curve fit that spans all cells with an explicit influence on the interface value. Each FWT is evaluated at the beginning of a timestep. A very simple way to satisfy this requirement in one space dimension is to run an FWT fit centered at each cell interface. Such an approach is not readily extended into multidimensional simulations using simplex elements. In the present formulation, a domain is divided into elements. (See Fig. 11.) Each element contains $N=2^{p}$ cells. A single FWT curve fit is derived for each dependent variable spanning the element. In general, a fourth-order accurate formulation of inviscid flux at cell face $i+1 / 2$ requires support from cells $i-2$ to $i+3$. The interior cell walls from $\mathrm{N} / 4+1$ to $3 \mathrm{~N} / 4$, indicated by the upper black brackets in Fig. 11, are all defined relative to the elemental FWT fit. The remaining cell walls within N/4

cells of an elemental boundary, indicated by the lower red bracket in Fig. 11, are defined relative to a blended FWT fit spanning the interior elemental boundaries.

The model equation system is given by

$$
\frac{\partial \mathbf{q}}{\partial t}+\frac{\partial \mathbf{f}}{\partial x}+\frac{\partial \mathbf{h}}{\partial x}+s(\mathbf{q}, x, t)=0
$$




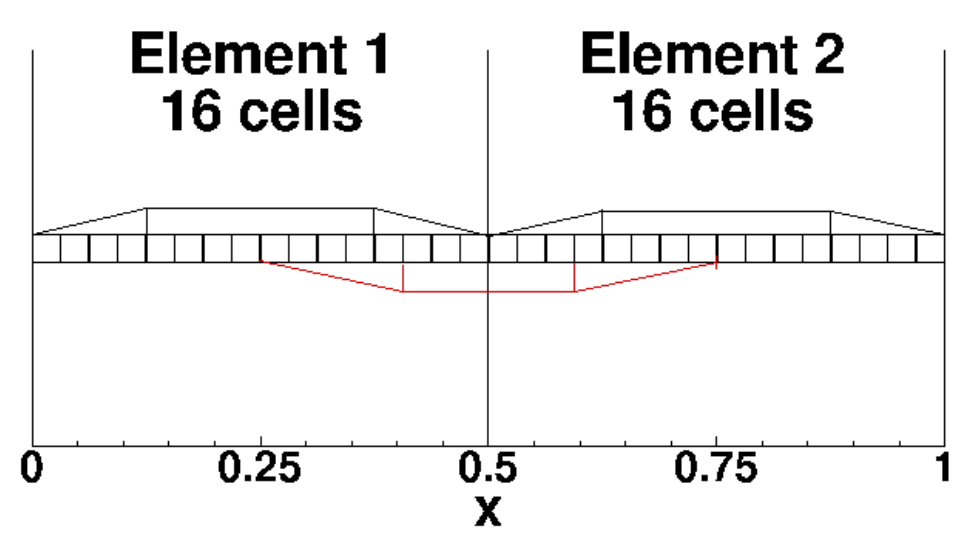

Figure 11. Schematic of the stencils used to define the inviscid flux across cell boundaries. Each element is composed of 16 cells. An FWT fit is applied across each element. The upper black bracket defines the cell boundaries derived from the elemental FWT fit. A blending FWT fit is applied across element boundaries. The lower red bracket defines the cell boundaries derived from the blended FWT fit.

A fourth-order Runge Kutta method is used to advance the solution in time. ${ }^{23}$ Let

$$
\mathbf{F}(x, t, \mathbf{q})=-\left[\frac{\partial \mathbf{f}}{\partial x}+\frac{\partial^{2} \mathbf{f}_{v}}{\partial x^{2}}+\mathbf{s}(\mathbf{q}, x, t)\right] .
$$

Then, given an initial distribution at time $t=t_{0}$, the solution at $t=t_{0}+\Delta t$ is given by

$$
\mathbf{q}\left(x, t_{0}+\Delta t\right)=\mathbf{q}\left(x, t_{0}\right)+\frac{1}{6}\left(\boldsymbol{\Omega}_{1}(x)+2 \boldsymbol{\Omega}_{2}(x)+2 \boldsymbol{\Omega}_{3}(x)+\boldsymbol{\Omega}_{4}(x)\right)
$$

where

$$
\begin{aligned}
& \boldsymbol{\Omega}_{1}(x)=\Delta t \mathbf{F}\left(x, t_{0}, \mathbf{q}\left(x, t_{0}\right)\right) \\
& \boldsymbol{\Omega}_{2}(x)=\Delta t \mathbf{F}\left(x, t_{0}+\frac{1}{2} \Delta t, \mathbf{q}\left(x, t_{0}\right)+\frac{1}{2} \boldsymbol{\Omega}_{1}(x)\right) \\
& \boldsymbol{\Omega}_{3}(x)=\Delta t \mathbf{F}\left(x, t_{0}+\frac{1}{2} \Delta t, \mathbf{q}\left(x, t_{0}\right)+\frac{1}{2} \boldsymbol{\Omega}_{2}(x)\right) \\
& \boldsymbol{\Omega}_{4}(x)=\Delta t \mathbf{F}\left(x, t_{0}+\Delta t, \mathbf{q}\left(x, t_{0}\right)+\boldsymbol{\Omega}_{3}(x)\right)
\end{aligned}
$$

A conventional, fourth-order accurate formulation in space for the inviscid (or viscous) flux terms are defined by

$$
\left(\frac{\partial \mathbf{f}}{\partial x}\right)_{i}=\left(27\left(\mathbf{f}_{i+1 / 2}-\mathbf{f}_{i-1 / 2}\right)-\left(\mathbf{f}_{i+3 / 2}-\mathbf{f}_{i-3 / 2}\right)\right) /(24 \Delta x)
$$

When discontinuities are detected in the FWT they cannot be located with any greater precision than one cell size. The detection algorithm simply informs that a discontinuity has been detected between cell center $i$ and cell center $i+1$. For convenience, the discontinuity is assigned a location at the cell interface, $i+1 / 2$. In this case, the limiting value of $f$ when approaching the cell interface from below, $f_{i+1 / 2}^{-}$, is not equal to the limiting value of $f$ when approaching the cell interface from above, $f_{i+1 / 2}^{+}$. It also follows in this case that the variation of $f$ between $x_{i-1 / 2}^{+}$and $x_{i+1 / 2}^{-}$is continuous. Equation 24 is therefore modified to account for the possibility of a discontinuity at a cell interface as follows.

$$
\left(\frac{\partial \mathbf{f}}{\partial x}\right)_{i}=\left(26\left(\mathbf{f}_{i+1 / 2}^{-}-\mathbf{f}_{i-1 / 2}^{+}\right)-\left(\mathbf{f}_{i+3 / 2}^{-}-\mathbf{f}_{i+1 / 2}^{+}\right)-\left(\mathbf{f}_{i-1 / 2}^{-}-\mathbf{f}_{i-3 / 2}^{+}\right)\right) /(24 \Delta x)
$$


Even though fourth-order spatial accuracy is lost in the presence of a discontinuous jump (except for some special cases), it is expected that this formulation will preclude the need for additional limiting or adjustment of stencils. This formulation is used for the advection and Burgers' equation test cases that follow. A second-order accurate formulation is expressed by

$$
\left(\frac{\partial \mathbf{f}}{\partial x}\right)_{i}=\left(\mathbf{f}_{i+1 / 2}^{-}-\mathbf{f}_{i-1 / 2}^{+}\right) /(\Delta x)
$$

Inviscid flux terms $\mathbf{f}_{i+1 / 2}^{ \pm}$will be defined relative to the FWT fits in Sec B. Viscous flux terms $\mathbf{h}_{i+1 / 2}$ are defined in $\mathrm{C}$.

\section{A. Preservation of Discontinuities}

The right and left state of a discontinuity in an element may be defined in one of two ways. If the FWT is a good fit to the data spanning an element, then the right and left states across a cell wall $x^{*}=x_{i+1 / 2}$ are computed from Eq. 9 with $x=x^{*} \pm \epsilon \Delta x$, where $\epsilon=0.0001$ herein. A good fit is defined as

$$
L_{1}=\frac{\sum_{n=1}^{2^{p}}\left|q^{p}\left(x_{i}\right)-\tilde{q}^{p}\left(x_{i}\right)\right|}{2^{p}}<L_{1, \text { target }}
$$

where $L_{1, \text { target }}=0.01$ for the Euler equations. Note that the Euler equations use FWT fits of the primitive variables, $\mathbf{q}^{p}$. (This conditional was never needed for the simpler advection and Burgers' sample problems.) If the FWT fit fails this test, then an exact Riemann solver ${ }^{9}$ is applied across every cell wall in the element and the right and left states are defined by the values at adjacent cell centers.

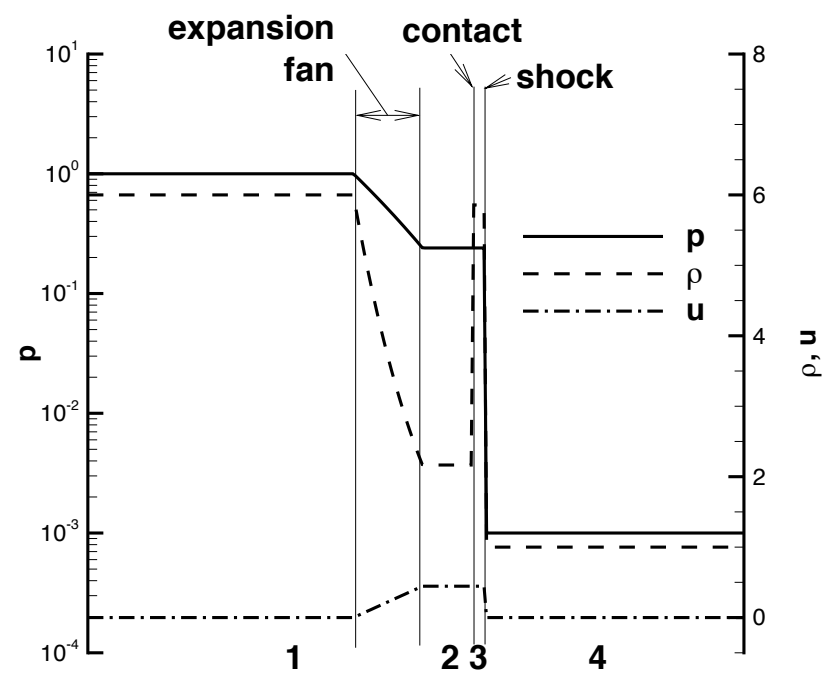

Figure 12. Riemann problem schematic example: given an initial state 1 and state 4 on either side of a diaphragm, three waves are formed upon the abrupt removal of the diaphragm. These waves include a shock or expansion fan moving to the left separating intermediate state 2 from state 1 and a shock or expansion fan moving to the right separating intermediate state 3 from state 4 . A contact discontinuity separates intermediate states 2 and 3 . The variation of pressure, density, and velocity are shown, with pressure and velocity continuous across a contact discontinuity.

In general, a discontinuity in the Euler equations decomposes into three waves as defined by the Riemann problem illustrated in Fig. 12. The algorithm for detecting and tracking these 
discontinuities is represented in the flow chart of Fig 13 with additional details as follows. An exact Riemann solver ${ }^{9}$ is used to determine the intermediate states 2 and 3 given the left and right states, 1 and 4, respectively, from Fig. 12. The Riemann solver will also provide the wave velocities of any shock or expansion fan separating states 1 from 2 (left running) and 3 from 4 (right running) as well as the contact surface velocity separating states 2 and 3. For the purpose of feature detection in this work, a detection threshold is defined for a shock as a function of the pressure ratio across the wave, $\left(p_{\text {high }} / p_{\text {low }}\right)_{t h r}$. In like manner, a detection threshold is defined for contact discontinuities as a function of the density ratio, $\left(\rho_{\text {high }} / \rho_{\text {low }}\right)_{t h r}$. Expansion fans are not preserved as discontinuities. Feature tracking requires that the bounding states and velocity of the leftmost detected wave be stored as $\mathbf{q}_{L}^{p}, \mathbf{q}_{L, \text { inner }}^{p}$, and $V_{L}$. In like manner, the bounding states and velocity of the rightmost detected wave are stored as $\mathbf{q}_{R}^{p}, \mathbf{q}_{R \text {,inner }}^{p}$, and $V_{R}$. A key element of this algorithm is to accept the fact that, even though a wave grouping as represented in Fig. 12 may be comprised of distinct waves after some small amount of time, it is not possible to observe that distinction in discretized space until at least one element of the group moves past a cell center. Consequently, an FWT detected discontinuity between any two cell centers must include the leftmost and rightmost states given by $\mathbf{q}_{L}^{p}, \mathbf{q}_{L, \text { inner }}^{p}, V_{L}, \mathbf{q}_{R}^{p}, \mathbf{q}_{R, \text { inner }}^{p}$, and $V_{R}$. The detected discontinuities identified as a shock

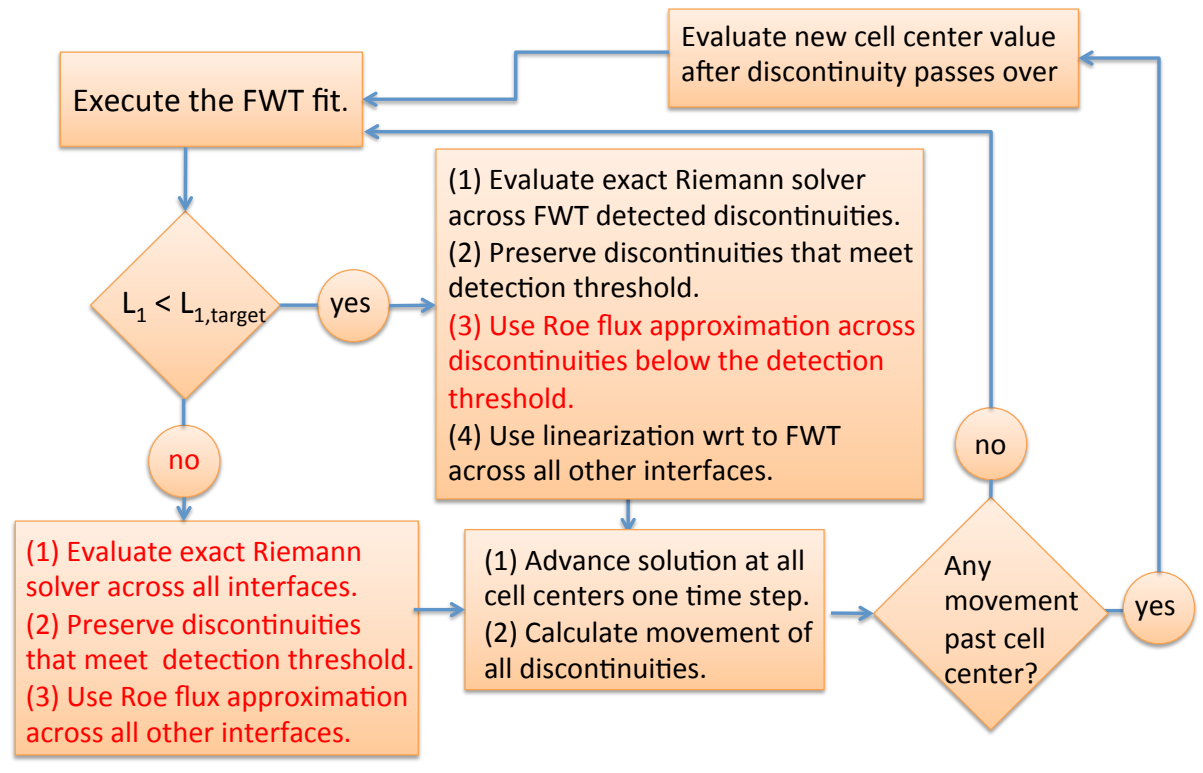

Figure 13. A flow chart for the detection and tracking of discontinuities. The logic proceeds from the upper left corner and is executed on every element in the domain. One cycle through the chart corresponds to one time step. Options in red text are only required for the Euler equations.

or contact surface serve as internal boundary conditions for the flow solver. Therefore, the finite volume formulation for any cell bordering a discontinuity will use

$$
\begin{aligned}
& \mathbf{f}_{i+1 / 2}^{-}=\mathbf{f}\left(\mathbf{q}_{L}^{p}\right) \\
& \mathbf{f}_{i+1 / 2}^{+}=\mathbf{f}\left(\mathbf{q}_{R}^{p}\right) .
\end{aligned}
$$

The detection algorithm starts afresh every time step. Shocks will appear or disappear based solely on the detection parameters ${ }^{19}$ used within the FWT and the threshold parameters, 
$\left(p_{\text {high }} / p_{\text {low }}\right)_{\text {thr }}$ and $\left(\rho_{\text {high }} / \rho_{\text {low }}\right)_{\text {thr }}$, filtering the Riemann solver. Still, it is necessary to maintain information regarding the previous location of a detected discontinuity so that its movement across the element may be computed. To enable shock movement, a data storage entity defined as a floater is created. Two floaters per element are allowed because the FWT (as currently configured) can only discern at most two discontinuities. Floaters are initialized to a null state. (See Fig. 14.) A newly detected discontinuity is assigned to a floater to enable its location $s$ relative to $x_{i+1 / 2}$ to be tracked. A detected discontinuity matches an existing floater location if it lies between the same two adjacent cell centers. If, after a new time step, a floater is not matched, then the feature disappears as a discontinuity and the cell interface is no longer treated as an internal boundary condition. Though not shown in the flow chart, floaters are free to cross element boundaries.

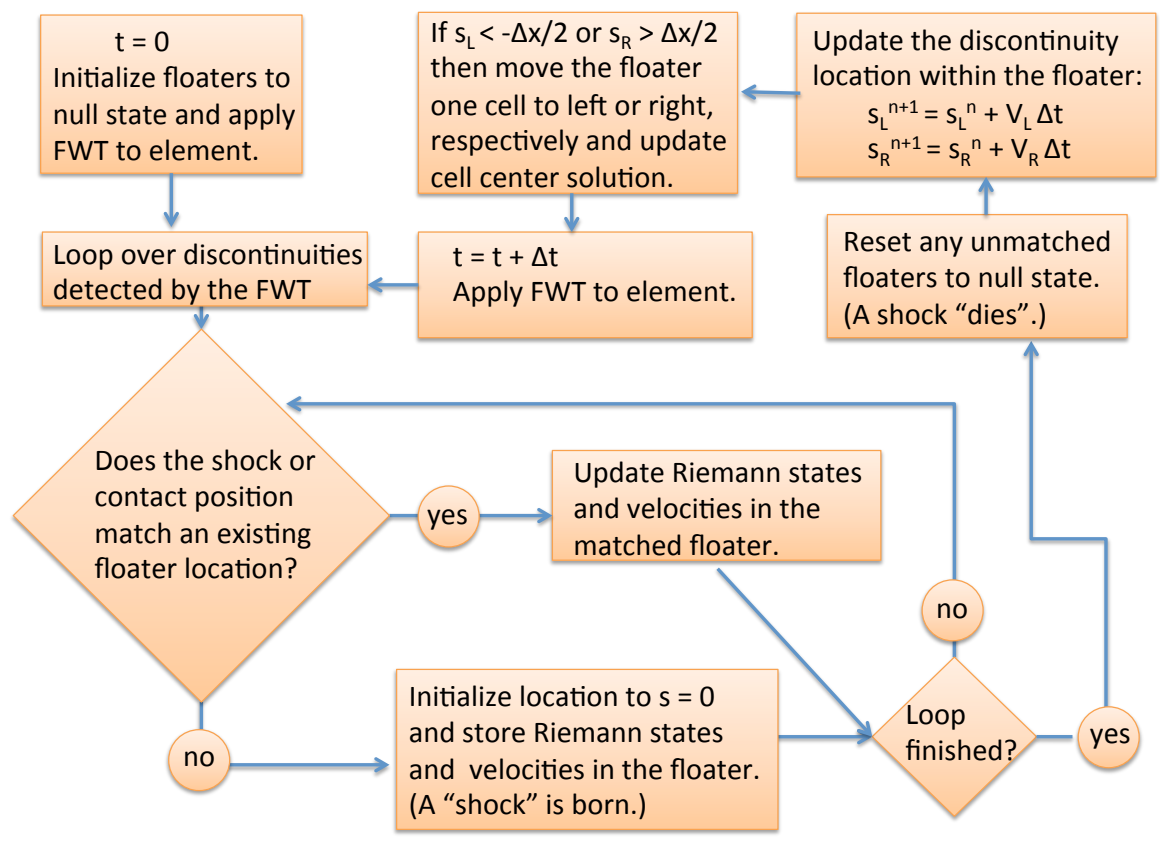

Figure 14. A flow chart for use of floaters, storage entities within an element that maintain data required for shock tracking. Logical flow starts from the upper left for each element.

In the case of the Euler equations, the FWT may detect a discontinuity that is too weak to register as either a shock or a contact surface. In this case, no effort is made to preserve the discontinuity as a distinct entity and a Roe's approximate Riemann solver is used to provide a definition of $\mathbf{f}_{i+1 / 2}$. Consequently, the flux for this transitional state between a clearly continuous and clearly discontinuous profile assigns, $\mathbf{f}_{i+1 / 2}^{+}=\mathbf{f}_{i+1 / 2}^{-}$.

\section{B. Inviscid Flux}

The previous section defines $\mathbf{f}_{i+1 / 2}^{ \pm}$for cases where a discontinuity is detected by the FWT between cells $i$ and $i+1$. In this section, a continuous variation is expected across the interface and the FWT is utilized to provide enhanced accuracy as discussed previously in Sec. IV and illustrated in Figs. 5 and 6 .

The flux Jacobian is diagonalized in the usual way in Eq. 30.

$$
\frac{\partial \mathbf{f}}{\partial \mathbf{q}}=\mathbf{A}=\mathbf{R}^{-1} \boldsymbol{\Lambda} \mathbf{R}
$$


The conversion from primitive to characteristic variables is defined relative to the FWT fit at cell centers $i$ using the matrix defined in Eq. 31. Differential contributions to the flux emanating from left and right running waves are evaluated with respect to the FWT fit at cell interfaces $i+1 / 2$ using the matrices defined in Eqs. 32 and 33.

$$
\begin{aligned}
\mathbf{R}_{i} & =\mathbf{R}\left(\tilde{\mathbf{q}}\left(x_{i}\right)\right) \\
\boldsymbol{\Lambda}_{i+1 / 2} & =\boldsymbol{\Lambda}\left(\tilde{\mathbf{q}}\left(x_{i+1 / 2}\right)\right) \\
\mathbf{R}_{i+1 / 2}^{-1} & =\mathbf{R}^{-1}\left(\tilde{\mathbf{q}}\left(x_{i+1 / 2}\right)\right)
\end{aligned}
$$

The difference between the actual value of the dependent variable $\mathbf{q}_{i}$ and the FWT fit to that same variable at cell center $x_{i}$ is given by Eq. 34. This differential represents a correction to the FWT value of the dependent variable at cell centers. The corresponding differential of the characteristic variable is defined in Eq. 35.

$$
\begin{aligned}
\delta \mathbf{q}_{i} & =\mathbf{q}_{i}-\tilde{\mathbf{q}}\left(x_{i}\right) \\
\delta \mathbf{q}_{i}^{\prime} & =\mathbf{R}_{i} \delta \mathbf{q}_{i}
\end{aligned}
$$

Note that the variation of these differential quantities is expected to be continuous and differentiable, even across discontinuities.

A fourth-order accurate interpolation formula is used to bring the cell-centered, differential corrections from the left (Eq. 36) and from the right (Eq. 37) to the cell interface at $i+1 / 2$,

$$
\begin{aligned}
\delta \mathbf{q}_{i+1 / 2}^{\prime+} & =L\left(\delta \mathbf{q}_{i+1}^{\prime}, \delta \mathbf{q}_{i}^{\prime}, \delta \mathbf{q}_{i-1}^{\prime}, \delta \mathbf{q}_{i-2}^{\prime}\right) \\
\delta \mathbf{q}_{i+1 / 2}{ }_{i+1}^{-} & =L\left(\delta \mathbf{q}_{i}^{\prime}, \delta \mathbf{q}_{i+1}^{\prime}, \delta \mathbf{q}_{i+2}^{\prime}, \delta \mathbf{q}_{i+3}^{\prime}\right)
\end{aligned}
$$

where the interpolation formula for equally spaced cells is given by Eq. 38 .

$$
L(a, b, c, d)=(5 a+15 b-5 c+d) / 16
$$

Discerning right and left running corrections to the flux at interface $i+1 / 2$ employs the eigenvalue and eigenvector matrices in Eqs. 39 and 40. The right and left corrections are finally applied to the FWT reference of flux $\tilde{\mathbf{f}}\left(\tilde{\mathbf{q}}\left(x_{i+1 / 2}\right)\right)$ in Eq. 41.

$$
\begin{aligned}
\delta \mathbf{f}_{i+1 / 2}^{+} & =\frac{1}{2} \mathbf{R}_{i+1 / 2}^{-1}\left[\boldsymbol{\Lambda}_{i+1 / 2}+\left|\boldsymbol{\Lambda}_{i+1 / 2}\right|\right] \delta \mathbf{q}_{i+1 / 2}^{++} \\
\delta \mathbf{f}_{i+1 / 2}^{-} & =\frac{1}{2} \mathbf{R}_{i+1 / 2}^{-1}\left[\boldsymbol{\Lambda}_{i+1 / 2}-\left|\boldsymbol{\Lambda}_{i+1 / 2}\right|\right] \delta \mathbf{q}_{i+1 / 2}^{--} \\
\mathbf{f}_{i+1 / 2} & =\tilde{\mathbf{f}}\left(\tilde{\mathbf{q}}\left(x_{i+1 / 2}\right)\right)+\delta \mathbf{f}_{i+1 / 2}^{+}+\delta \mathbf{f}_{i+1 / 2}^{-}
\end{aligned}
$$

\section{Viscous Flux}

Whereas the inviscid flux terms were defined relative to the FWT fit, the viscous terms are defined relative to $\mathbf{q}_{i}$. The inviscid formulation is designed to detect and preserve discontinuities. The viscous formulation, consistent with the physical nature of dissipation, must retain the ability to dissipate (spread out) the shock. The competing influence of these formulations on shock structure will be discussed further in the Burgers' equation examples that follow in Sec. B. The fourth-order accurate formulation of viscous flux on a cell wall is therefore defined by

$$
\mathbf{h}_{i+1 / 2}=\nu\left(\frac{\partial \mathbf{q}}{\partial x}\right)_{i+1 / 2}=\nu\left(27\left(\mathbf{q}_{i+1}-\mathbf{q}_{i}\right)-\left(\mathbf{q}_{i+2}-\mathbf{q}_{i-1}\right)\right) / 24 \Delta x .
$$


This formulation utilizes the identical stencil defined for the inviscid flux in Fig. 11. A modified version of Eq. 42 recognizes that the available resolution may be insufficient to resolve a shear layer. Equation 43 prevents formation of non-physical extrema across the discontinuity if the physical viscosity coefficient is too small.

$$
\begin{aligned}
\mathbf{h}_{i+1 / 2} & =\nu_{0}\left[\sum_{k=1}^{m} k a_{k} x_{i+1 / 2}^{k-1}+\left(27\left(\delta \mathbf{q}_{i+1}-\delta \mathbf{q}_{i}\right)-\left(\delta \mathbf{q}_{i+2}-\delta \mathbf{q}_{i-1}\right)\right) / 24 \Delta x\right] \\
& +\left(\nu-\nu_{0}\right)\left[\quad\left(27\left(\mathbf{q}_{i+1}-\mathbf{q}_{i}\right)-\left(\mathbf{q}_{i+2}-\mathbf{q}_{i-1}\right)\right) / 24 \Delta x\right]
\end{aligned}
$$

where $\nu_{0}=\min (\nu, \Delta x / 2)$ and the $a_{k}$ are the FWT derived curve fit coefficients of Eq. 9.

\section{Sample applications}

\section{A. Advection Equation}

The Advection equation is defined from the model system (Eq. 17) with $\mathbf{f}=c \mathbf{q}, \mathbf{h}=0(\nu=0)$, and $\mathbf{s}=0$ with $\mathbf{q}$ a scalar. The domain is given by $0 \leq x \leq 1$ and a periodic boundary condition is applied such that $\mathbf{q}(0, t)=\mathbf{q}(1, t)$. The wave speed $c=1$ is a constant and the matrices in Eqs. 31 - 33 are scalars, identically equal to 1 . Explicit time advancement is used with $\Delta t=\Delta x$. The exact solution is expressed with a transformation from $x$ and $t$ to $z$ by

$$
z= \begin{cases}x-c t & \text { for } \quad x \geq c t \\ 1-(|x-c t|-\operatorname{trun}(|x-c t|)) & \text { for } \quad x<c t\end{cases}
$$

so that $\mathbf{q}(z) \equiv \mathbf{q}(x, 0)$.

Results for the advection of a Gaussian profile are shown in Fig. 15. There are no discontinuities to be detected in this example. The computed profile is indistinguishable from the exact solution after 20 cycles across the domain in Fig. 15a for a simulation with 8 elements, $2^{6}=64$ cells per element $(p=6)$, using an FWT fit polynomial of degree $6(m=6)$. The $L_{1}$ error norm for several simulations relative to the exact solution after 20 cycles is presented in Fig. 15b, where $L_{1}=\sum \Delta x\left|\mathbf{q}\left(x_{i}, 0\right)-\mathbf{q}\left(z_{i}\right)\right|$. The total number of cells in a simulation equals the number of elements times the number of cells per element $\left(2^{p}\right)$. A four element simulation with $2^{p}$ cells per element has approximately the same error norm as an eight element simulation with $2^{p-1}$ cells per element. The slope of $\log _{10}\left(L_{1}\right)$ with respect to $p$ for simulations with the same number of elements equals -4 , indicating a fourth-order accurate simulation in space and time. The $\Delta$ symbols in Fig. 15b indicate results for which the FWT fit was circumvented by setting $\tilde{\mathbf{q}}_{i}$ to zero. This result shows a factor two reduction in the error when using the FWT fit (square symbols) as compared to a more conventional formulation (delta symbols), even for a case in which there are no discontinuities.

Results for the advection of a triangular profile that is $C_{0}$ continuous, but has $C_{1}$ discontinuities at $z=0.25,0.50$, and 0.75 , are shown in Fig. 16. The computed profile shows slight rounding of the corners after 20 cycles in Fig. 16a for a simulation with 8 elements, $2^{6}=64$ cells per element $(p=6)$, using an FWT fit polynomial of degree $6(m=6)$. The corresponding $L_{1}$ error norm for several simulations relative to the exact solution after 20 cycles is presented in Fig. 16b. The presence of $C_{1}$ discontinuities disrupts the fourth-order convergence observed for the previous case. The approximate order of convergence is 1.5. Simulations with and without the FWT fit are essentially identical - suggesting that discontinuities in slope may be an additional feature worth detecting in future work.

Results for the advection of a cosine profile superposed on a Heaviside function are shown in Fig. 17. This is the first example with a $C_{0}$ discontinuity included in the initial profile. The 


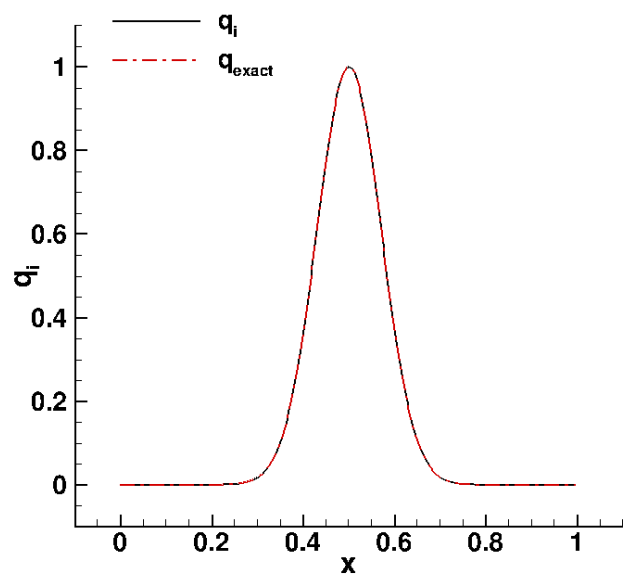

(a) $p=6, m=6,20$ cycles

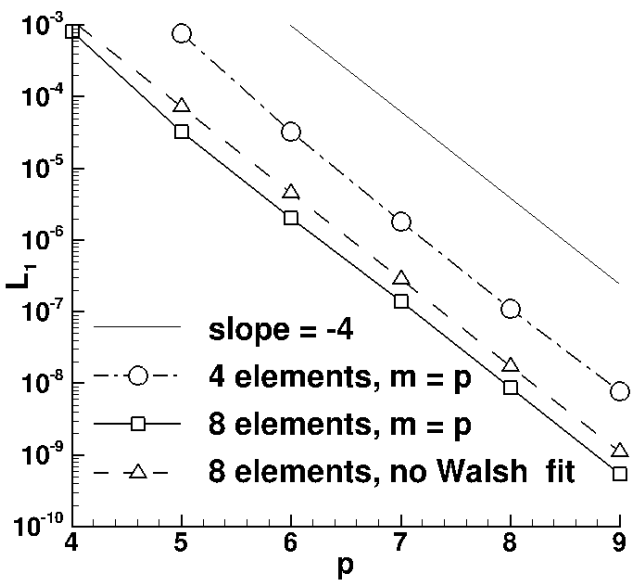

(b) error norm

Figure 15. Advection of Gaussian profile $f_{i}=\exp \left(-10\left(z_{i}-0.5\right)^{2}\right)$ after 20 cycles across the domain.

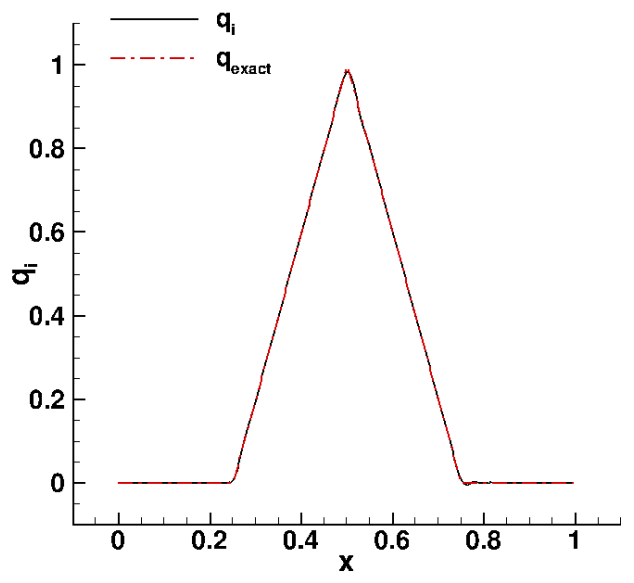

(a) $p=6, m=6,20$ cycles

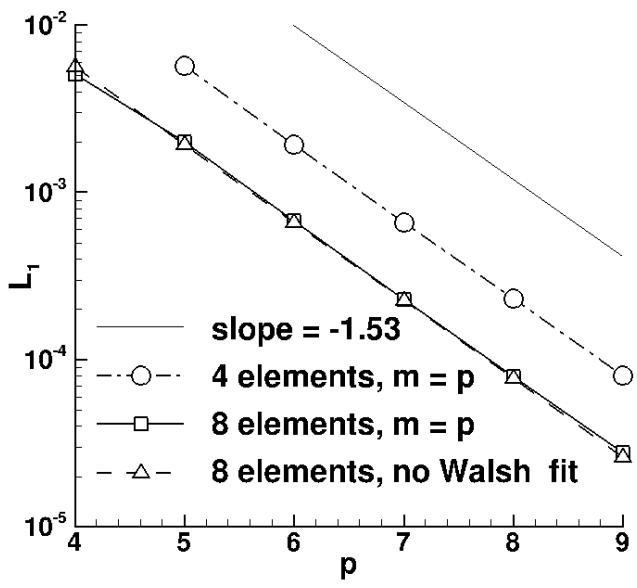

(b) error norm

Figure 16. Advection of triangle profile $\left.f_{i}=1-4 \mid z_{i}-0.5\right) \mid$ for $0.25 \leq z_{i} \leq 0.75$ and $f_{i}=0$ for $z_{i}<0.25$ or $z_{i}>0.75$ after 20 cycles across the domain.

discontinuity should advect to the right with speed equal to one. If the discontinuity is dissipated by the numerics in the solution of the advection equation, then there is no restorative action to resharpen the discontinuity - all characteristics have wave speed equal to one. Consequently, feature detection is exploited here to preserve the magnitude of the detected discontinuity and to move it with the wave speed, as outlined in Fig. 14. In this simple advection case, there is only a right running wave with wavespeed $V_{R}=1$. When the discontinuity crosses a cell center $x_{i}$, the cell is updated according to the translated FWT curve fit given by Eq. 9. (Note that this update algorithm is simpler than that described earlier; ${ }^{19}$ however, the results are nearly identical.)

This tracking algorithm effectively preserves the discontinuity through 20 cycles across the 


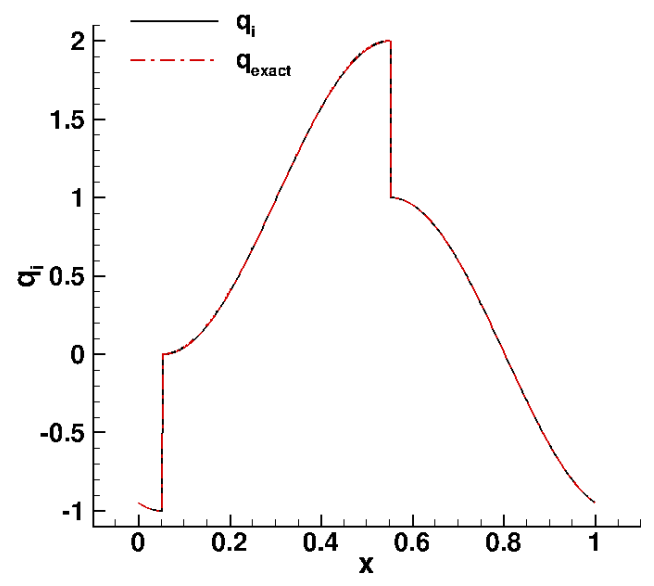

(a) $p=7, m=5,14.55$ cycles

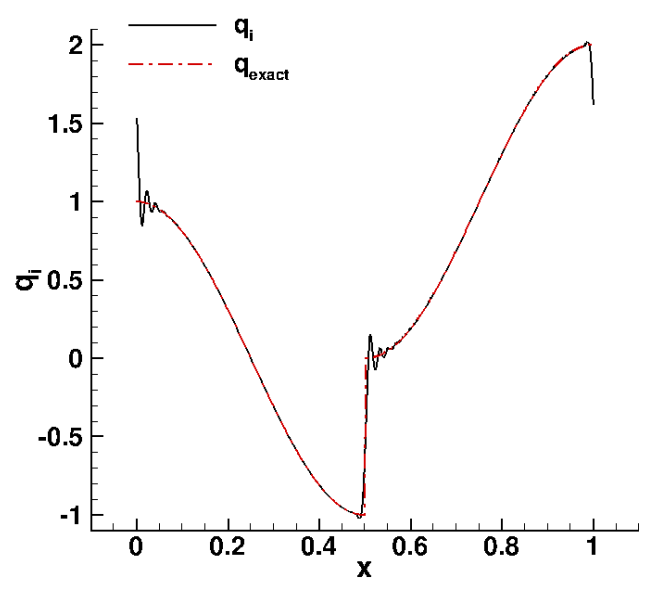

(c) no tracking, $p=7, m=5,20$ cycles

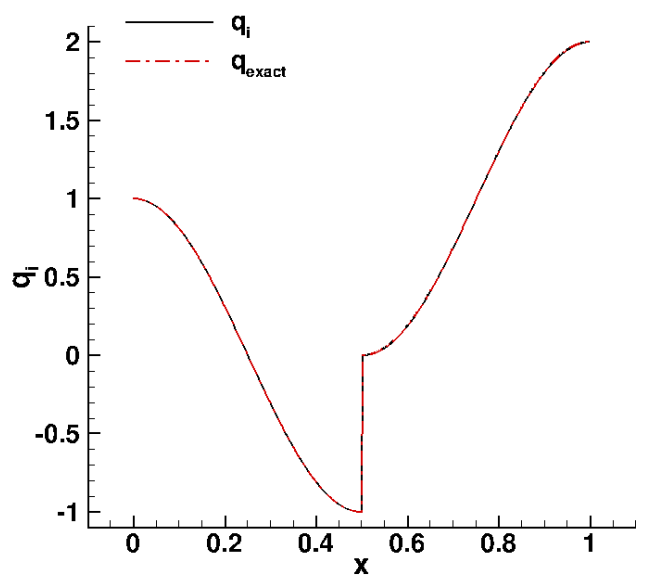

(b) $p=7, m=5,20$ cycles

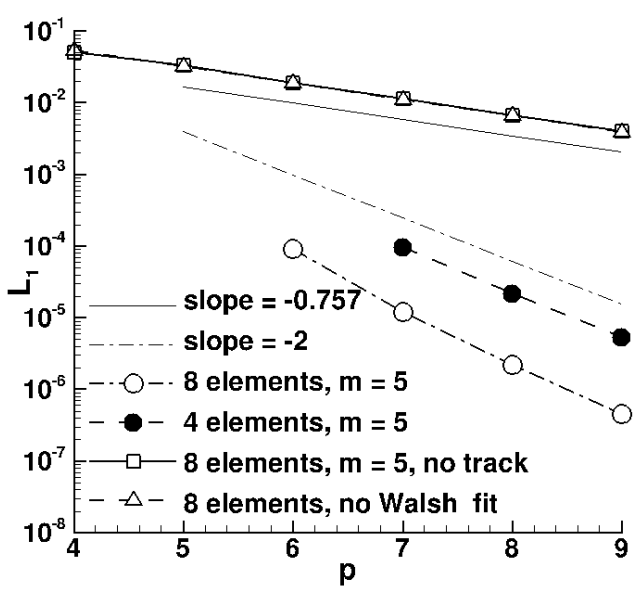

(d) error norm

Figure 17. Advection of cosine profile with a jump, $f_{i}=\cos \left(2 \pi z_{i}\right)+H\left(z_{i}, 0.5\right)$ for $0 \leq z_{i} \leq 1$.

domain, as seen in Fig. 17a and b. If the tracking algorithm is not engaged and a conventional limiter is not enforced, then ringing across the discontinuity is evident in Fig. 17c.

The error norms for several simulations varying the number of elements spanning the domain and the order of the FWT fit in each element are presented in Fig. 17d. Engaging the discontinuity tracking algorithm provides orders of magnitude more accurate results than without tracking and provides a convergence rate approaching third-order in space and time. It is important to note that the tracking algorithm diverges for $m>5$. It appears that the extra degree of freedom in the FWT fit enables waviness across the detected discontinuity that amplifies with increasing time.

\section{B. Burgers' Equation}

Burgers' equation is defined from the model system (Eq. 17) with $\mathbf{f}=\frac{1}{2} \mathbf{q}^{2}, \mathbf{h}=\nu \frac{\partial \mathbf{q}}{\partial x}$, and $\mathbf{s}=0$ with $\mathbf{q}$ a scalar. The domain is given by $-1 \leq x \leq 1$ and fixed boundary conditions are applied such that $\mathbf{q}(-1, t)=1$ and $\mathbf{q}(1, t)=-1$. The initial condition is given by $\mathbf{q}(x, 0)=-x$. The solution 
evolves to a steady state given by $\mathbf{q}(x)=C \tanh \left(\frac{C x}{2 \nu}\right)$. The constant $C$ is defined to recover the boundary values. In the limit $\nu \rightarrow 0, \mathbf{q}(x)=1-2 H(x, 0)$, where the Heaviside function was defined in Eq. 10. The matrices in Eqs. $31-33$ are: $\mathbf{R}_{i}=[1], \boldsymbol{\Lambda}_{i+1 / 2}=\left[\tilde{\mathbf{q}}_{i+1 / 2}\right]$, and $\mathbf{R}_{i+1 / 2}^{-1}=[1]$. Achieving high-order spatial accuracy in these simulations is simplified by using the value of the steady-state analytic solution for any formulation that requires cell center information beyond the boundaries.

In this example, a detected discontinuity between cells $i$ and $i+1$ has a single wave speed approximated by $V=\left(q\left(x_{i}\right)+q\left(x_{i+1}\right) / 2\right.$. When the discontinuity crosses an adjacent cell center, the cell is updated according to the translated FWT curve fit give by Eq. 9. (Again, this update algorithm is simpler than that described earlier; ${ }^{19}$ however, the results produce nearly identical results in the steady state.)

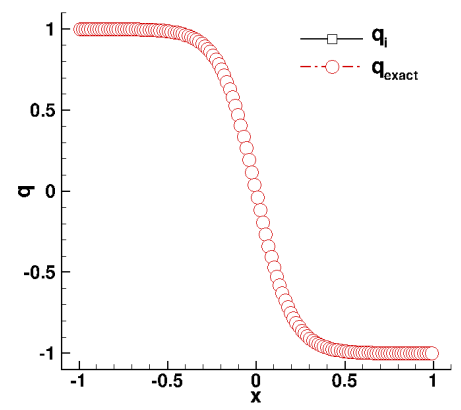

(a) $\nu=0.100, \Delta x / \nu=0.15625, n=$ 8

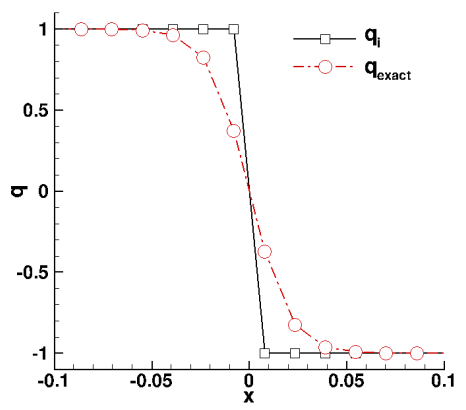

(b) $\nu=0.010, \Delta x / \nu=1.5625, n=8$, detail at shock

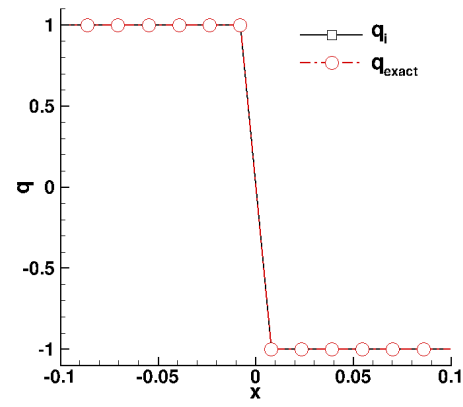

(c) $\nu=0.001, \Delta x / \nu=15.625, n=8$, detail at shock

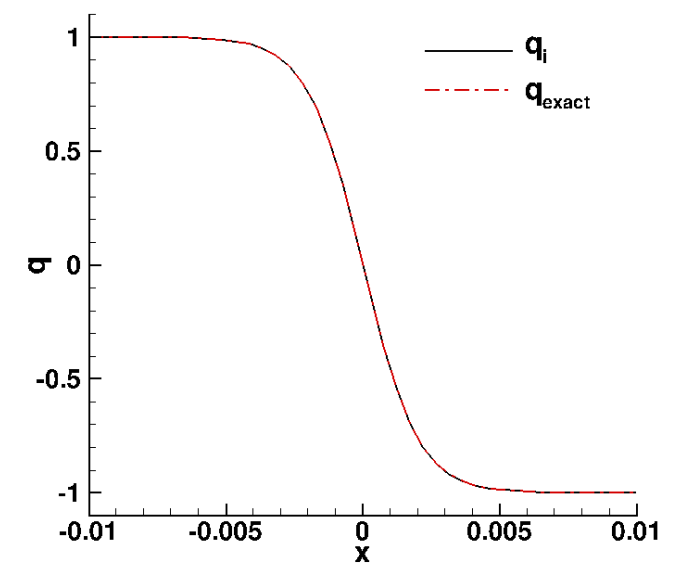

(d) $\nu=0.001, \Delta x / \nu=0.48828125, n=256$, detail at shock

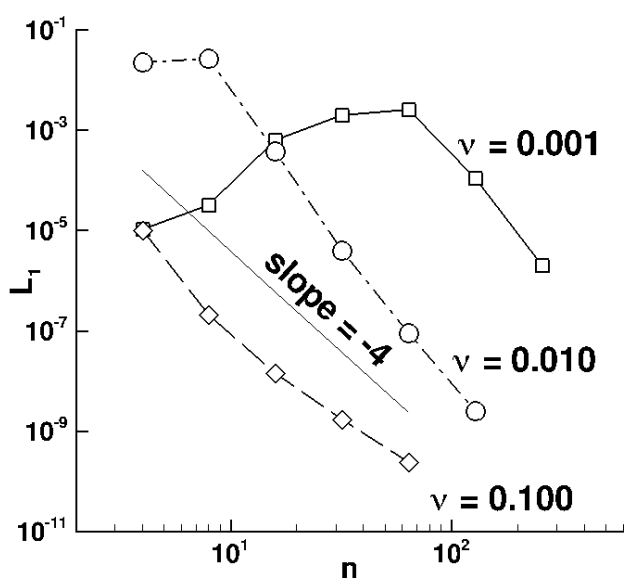

(e) error norm

Figure 18. Solution to Burgers' equation as a function of $\nu$ and number of elements $n$ with $p=4$ (16 cells per element) and $m=4$ (fourth-order FWT fit) including shock detection.

Results for the Burgers' equation simulations are shown in Fig. 18. Symbols in Figs. 18(ac) indicate cell center locations. Three diffusivity coefficients covering three orders of magnitude $(\nu=0.100,0.010$, and 0.001$)$ are considered. When $\Delta x / \nu<\mathrm{O}(1)$, the simulated shock is well resolved and when $\Delta x / \nu>\mathrm{O}(1)$, the simulated shock is unresolved. All of the $\nu=0.100$ simulations 
are well resolved. For example, Fig 18a, shows a well resolved shock with 8 elements and 16 cells per element. The $L_{1}$ norm for $\nu=0.100$ in Fig. 18e shows a monotone decrease with slope less than -4, indicating fourth-order accuracy. The $\nu=0.010$ simulations for $n<16$ have $\Delta x / \nu=\mathrm{O}(1)$. In these cases, the exact solution has only a few cells resolving the jump and the simulated solution either detects a shock with a jump across a single cell (as in Fig. 18b) or it places a discontinuity free FWT fit across the transition that tends to be sharper than the exact solution. The detection threshold $\left(h^{*}\right)^{19}$ for a discontinuity is set to 0.5 for these simulations; larger values tend to favor a smoother transition but may allow some small overshoot in the simulated profile. As the number of elements $n$ increases, the value of $\Delta x / \nu$ becomes less than 1 and fourth-order accuracy is evident in Fig. 18e. The $\nu=0.001$ simulations for $n<32$ have $\Delta x / \nu<\mathrm{O}(1)$. In this case, both the exact solution and the simulated solution show a jump across a single cell as shown in Fig. 18c. When $n>32$, the ratio $\Delta x / \nu$ becomes less than order 1 and the transition is well resolved, as shown in Fig. 18d. The $L_{1}$ norm shows fourth-order accuracy in Fig. 18e for this range with $n>32$. There is no clearly defined order of accuracy for smaller $n$; very small errors in the detected jump magnitude tend to keep the error norm of order $10^{-2}$. Numerical experiments indicate divergence for cases where the FWT polynomial has degree $m>4$ and $\Delta x / \nu>=\mathrm{O}(1)$. As with the advection equation, it appears that the extra degree of freedom in the FWT fit enables waviness across the detected discontinuity that amplifies with increasing time.

\section{Quasi-One-Dimensional, UnsteadyFlow}

The quasi-one-dimensional nozzle and shock tube problems are defined from the model system (Eq. 17) with $\mathbf{q}=[\rho A, \rho u A, \rho E A]^{T}, \mathbf{f}=\left[\rho u A, \rho u^{2} A+p A, \rho u H A\right]^{T}, \mathbf{h}=\nu \Delta x \frac{\partial \mathbf{q}}{\partial x}$, and $\mathbf{s}=\left[0,-p \frac{\partial A}{\partial x}, 0\right]^{T}$ where primitive, dimensionless variables are density $(\rho)$, velocity $(u)$, pressure $(p)$, and internal energy $(e)$. The total energy $(E)$ is defined $E=e+u^{2} / 2$ and total enthalpy $(H)$ is defined $H=E+p / \rho$. The equation of state for a perfect gas is $p=\beta \rho e$ where the ratio of specific heats for air as a perfect gas is $\gamma=1.4$ and $\beta=\gamma-1$. The speed of sound is $c=\sqrt{\gamma p / \rho}$. The matrices in Eqs. $31-33$ are defined $\boldsymbol{\Lambda}_{i+1 / 2}=[\tilde{u}-\tilde{c}, \tilde{u}, \tilde{u}+\tilde{c}]_{i+1 / 2}^{D}$ and

$$
\mathbf{R}_{i}=\left[\begin{array}{ccc}
1 & 1 & 1 \\
\tilde{u}-\tilde{c} & \tilde{u} & \tilde{u}+\tilde{c} \\
\tilde{H}-\tilde{u} \tilde{c} & \tilde{u}^{2} / 2 & \tilde{H}+\tilde{u} \tilde{c}
\end{array}\right]_{i} \quad \mathbf{R}_{i+1 / 2}^{-1}=\frac{1}{2 \tilde{c}^{2}}\left[\begin{array}{ccc}
\frac{1}{2} \beta \tilde{u}^{2}+\tilde{u} \tilde{c} & -\tilde{c}-\beta \tilde{u} & \beta \\
2 \tilde{c}^{2}-\beta \tilde{u}^{2} & 2 \beta \tilde{u} & -2 \beta \\
\frac{1}{2} \beta \tilde{u}^{2}-\tilde{u} \tilde{c} & \tilde{c}-\beta \tilde{u} & \beta
\end{array}\right]_{i+1 / 2}
$$

A superscript * indicates a dimensional quantity. Pressure and enthalpy are nondimensionalized by the left state initial conditions; consequently $p_{\text {ref }}=1$ and $H_{r e f}=1$. Density is nondimensionalized by $\rho^{*}=p_{\text {ref }}^{*} / H_{\text {ref }}^{*}$ so that $\rho_{\text {ref }}=\gamma /(\gamma-1)$. Velocity is nondimensionalized by $\sqrt{H_{r e f}^{*}}$.

The detection threshold ${ }^{19}\left(h^{*}\right)$ for a discontinuity is set to 0.3 for these Euler equation simulations. The acceptance threshold for a shock (see Fig. 13) is $\left(p_{\text {high }} / p_{\text {low }}\right)_{t h r}=1.05$ and for a contact discontinuity is $\left(\rho_{\text {high }} / \rho_{\text {low }}\right)_{t h r}=1.15$. If these parameters are set too high, then the algorithm will fail to detect some weaker but real discontinuous flow features. If these parameters are set too low, then false positives for the presence of a discontinuity may be produced that tend to degrade accuracy and/or stability.

\section{Nozzle}

The nozzle area distribution $A(x)$ used herein is defined

$$
A(x)=A_{\text {throat }}+3(1-\cos (\pi x)) \quad-1 \leq x \leq 1
$$


A nozzle schematic with boundary conditions is shown in Fig. 19. Two inflow boundary conditions at $x=x_{a}=-1$ match total enthalpy and isentropic flow from the plenum. A single outflow boundary condition at $x=x_{b}=1$ matches pressure at the exit plane.

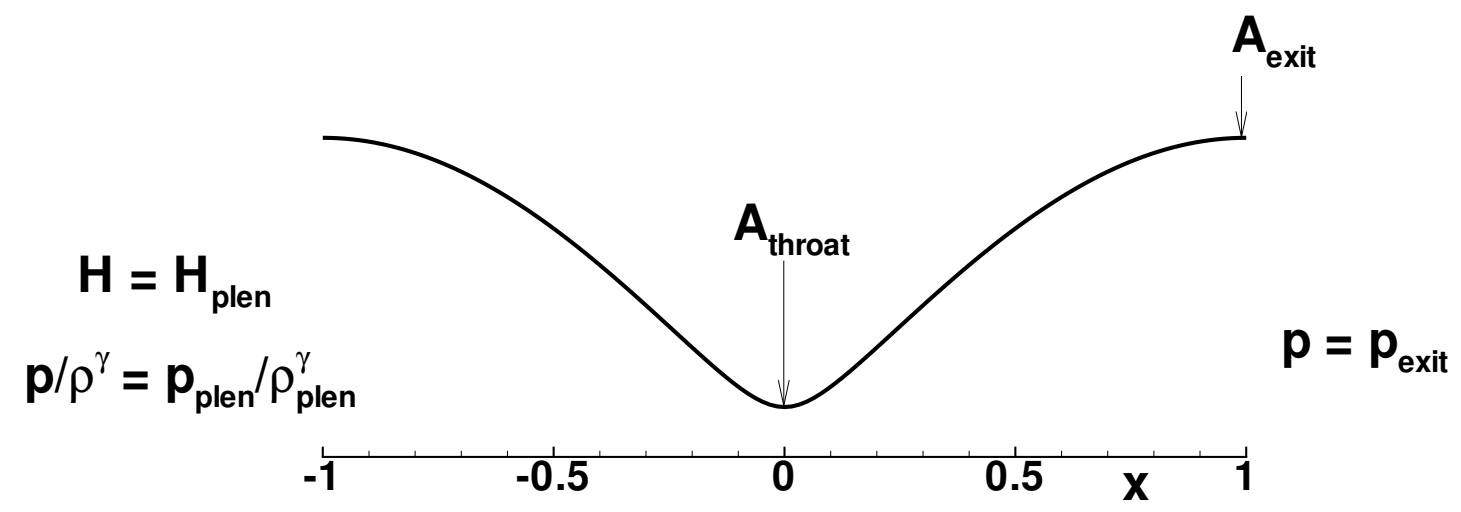

Figure 19. Schematic of nozzle geometry and boundary conditions.

Analytic solutions to nozzle flow can be defined as a function of area ratio. ${ }^{25}$ The nozzle has $A_{\text {throat }}=1$ and $A_{\text {exit }}=7$. If $1>p_{\text {exit }}>0.9951909$, then the flow throughout this nozzle is subsonic. If $0.9951909>p_{\text {exit }}>0.180148$, then the flow is sonic at the throat and a shock stands between the throat and the exit. The initial condition for all cases corresponds to a diaphragm at the nozzle throat separating plenum conditions from exit conditions with zero velocity and $\left(\rho_{\text {exit }} / \rho_{\text {ref }}\right)^{\gamma}=p_{\text {exit }} / p_{\text {ref }}$.

The test problem, with results at different times plotted in Fig. 20, sets $p / p_{\text {ref }}=0.2, \gamma=1.4$ and produces a standing shock near the exit with Mach number, $M=3.438$ and a pressure ratio across the shock equal to 13.6 in the steady-state limit. The lines in this figure indicate the analytic steady-state limit solution. The symbols indicate cell center values from the simulation. The initial profile (Fig. 20 a) spawns a left moving expansion and right moving shock shortly after the diaphragm bursts (Fig. 20 b). A clearly discernible contact surface is not evident in the density distribution for this condition. At $t=0.646$ the initial shock is near the exit plane and a steepening of the flow profile is evident in Fig. 20 c. In the next frame (Fig. $20 \mathrm{~d}$ ), the steepening profiles transform to a right moving shock. The jump is recognized and preserved across a single cell by the FWT algorithm. By $t=2.66$ the initial shock has exited, an expansion moves back up the nozzle and the shock "born" upstream in Fig. $20 \mathrm{~d}$ has moved far downstream, just past its steady-state position in Fig. 20 e. As time progresses, the shock moves back upstream and settles at the correct steady-state position in Fig. $20 \mathrm{f}$.

The simulation in Fig. 20 used 16 elements across the domain $(n=16)$ with 16 cells per element $(p=4)$ and an FWT derived polynomial fit of degree $4(m=4)$. The steady-state solution for simulations $n=8,16,32$ had associated error norms $L_{1}=2.0610^{-4}, 8.3110^{-05}, 3.2210^{-05}$, respectively. This reduction in error norm indicates an order of accuracy equal to 1.36. The current algorithm makes no attempt to adjust the difference stencil based on the exact location of the discontinuity between the cells and so the net error is substantially weighted by this first-order treatment. Also, it was observed that a steady-state solution could be obtained without limiting the application of Eqs. 25 and 38 but the standing shock formed several cells upstream of the correct location. Consequently, this simulation was executed using Eq. 26 and a first-order extrapolation $(L(a, b, c, d)=b)$ in place of Eq. 38 whenever the stencil spanned a discontinuity. 


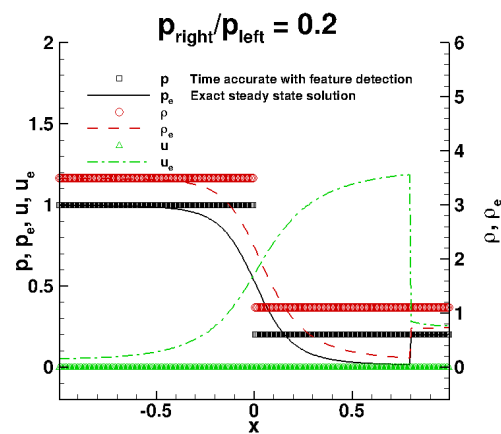

(a) Initial condition, $t=0$

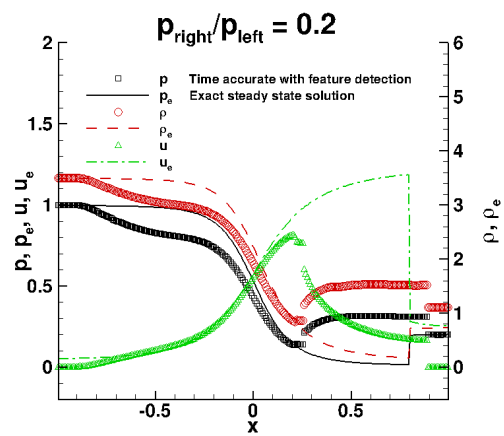

(d) Shock formation near $x=0.3$, $t=0.664$

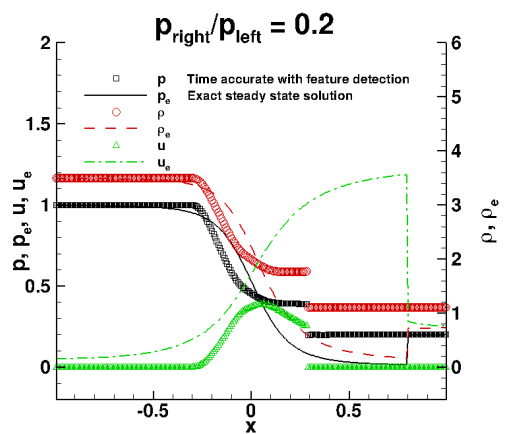

(b) Shortly after diaphragm bursts, $t=0.195$

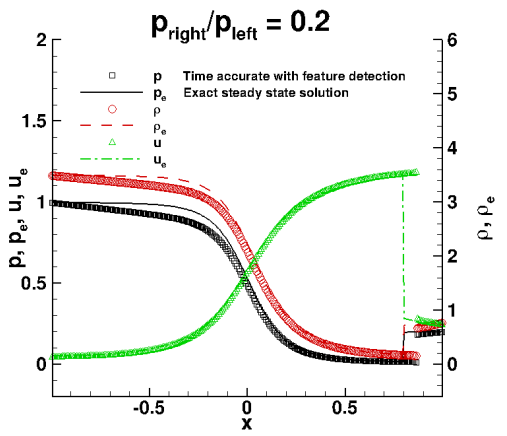

(e) Standing shock at position closest to exit, $t=2.66$

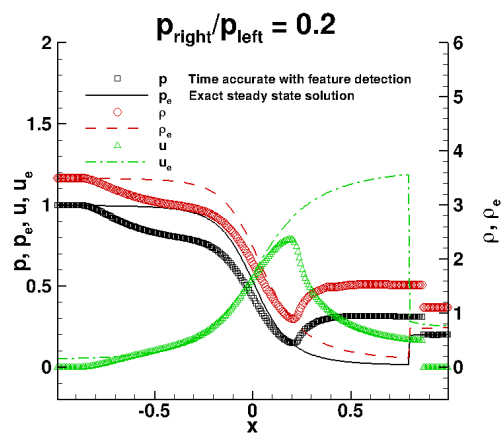

(c) Profile steepens near $x=0.3$ just prior to shock formation, $t=0.646$

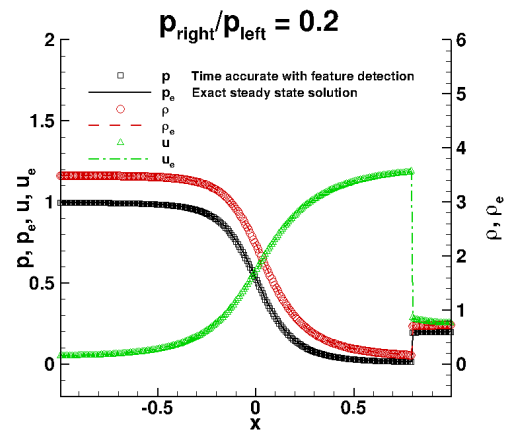

(f) Steady-state solution, $t=50$

Figure 20. Evolution of the nozzle flowfield. Diaphragm at throat separates plenum conditions $\left(p / p_{\text {ref }}=1, H / H_{r e f}=1\right)$ from exit condition $\left(p / p_{\text {ref }}=0.2\right)$. The exit density is initialized assuming an isentropic expansion to the exit pressure from the plenum conditions. Simulation parameters in this case are: $\Delta t / t_{r e f}=0.1 \Delta x, n=16, p=4$, and $m=4$.

\section{Shock Tube (Sod Problem)}

The shock tube has closed ends and a diaphragm at the midpoint that separates the left state $\left(p / p_{\text {ref }}=1, \rho / \rho_{\text {ref }}=6\right)$ from the right state $\left(p / p_{\text {ref }}=0.001, \rho / \rho_{\text {ref }}=1\right)$. An analytic solution to this problem following the diaphragm removal is easily derived from an exact Riemann solver up to the first reflection of the shock off the right closed end. This test case (frequently cited as the Sod problem ${ }^{26}$ ) produces a challenging contact discontinuity that follows the shock to the right. The simulation is executed with identical numerical parameters used in the previous nozzle case.

The first three frames of Fig. 21 show the profiles at $t / t_{r e f}=0,0.39$, and 0.781 , respectively. The lines show the time-accurate analytic solution assuming no reflections off the end walls. The symbols show the simulation results at the cell centers. Of particular interest is the density distribution shown by the red circles and dashed red line. The simulation preserves moving discontinuities at both the shock front and the contact discontinuity. Shock capturing techniques typically smear the shock over two to three cells and the contact discontinuity over many cells. The shock and the contact surface discontinuity in this simulation are generally within one cell of the exact solution. The error norm to the first reflection is shown in Fig. 22. The jaggedness of this plot is a consequence of the "quantized" movement of the discontinuities. The simulated discontinuity abruptly jumps from one cell interface to the next according to the logic in Fig. 14 whereas the analytic solution 
moves continuously across the domain.

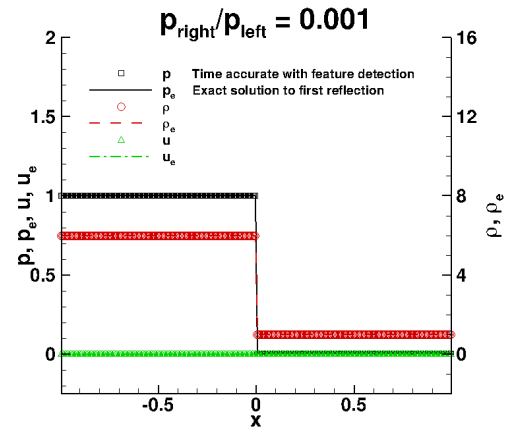

(a) Initial condition, $t / t_{\text {ref }}=0$

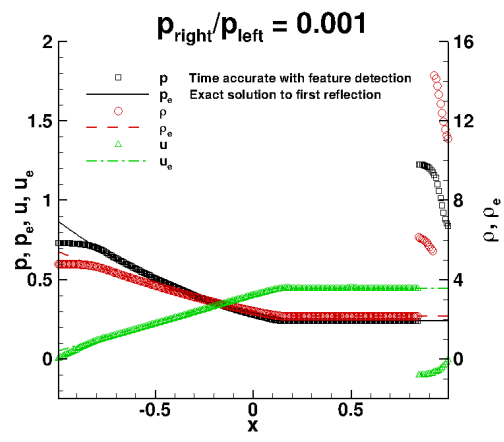

(d) $t / t_{r e f}=2.344$

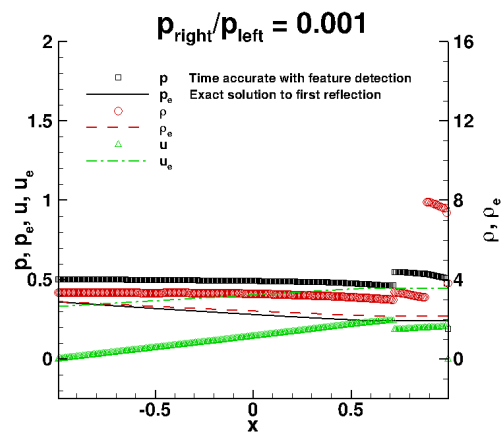

(g) $t / t_{\text {ref }}=11.328$

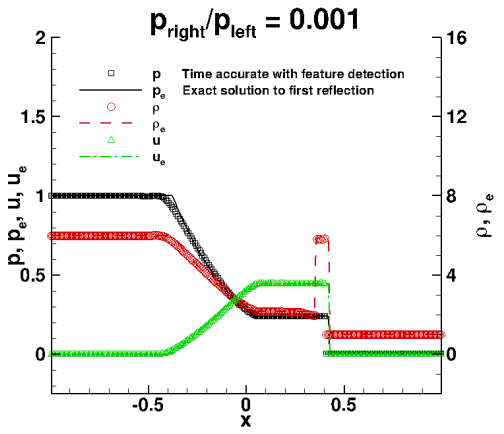

(b),$t / t_{\text {ref }}=0.782$

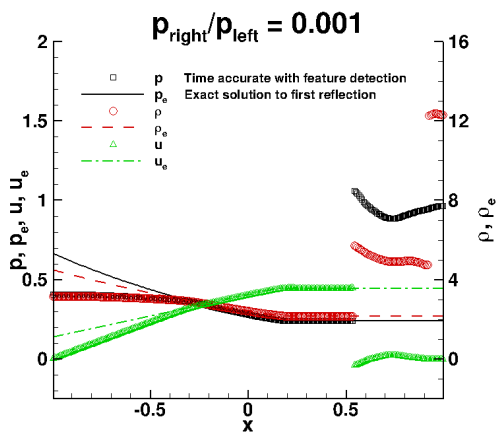

(e) $t / t_{\text {ref }}=3.126$

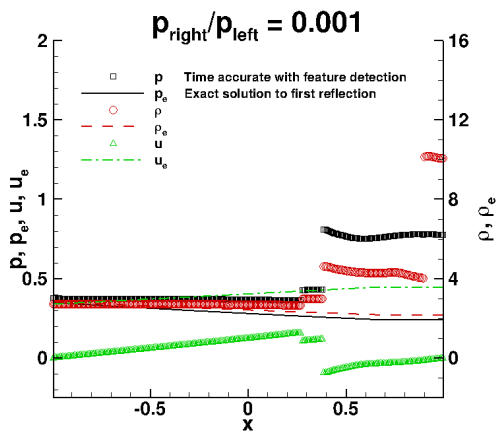

(h) $t / t_{\text {ref }}=12.890$

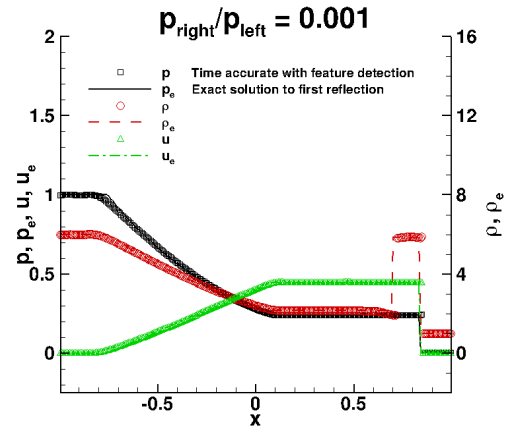

(c) $t / t_{\text {ref }}=1.562$

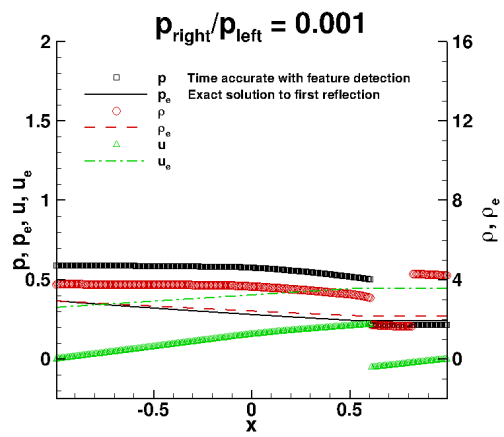

(f) $t / t_{r e f}=10.546$

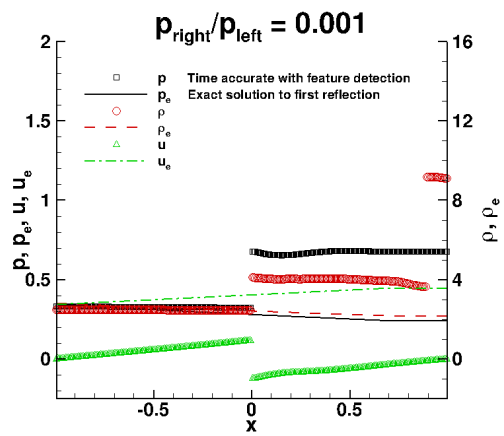

(i) $t / t_{r e f}=14.454$

Figure 21. Flow in closed tube after diaphragm at midpoint is removed. Diaphragm separates conditions at right $\left(p / p_{\text {ref }}=1, \rho / \rho_{\text {ref }}=6\right)$ from conditions at left $\left(p / p_{\text {ref }}=0.001, \rho / \rho_{\text {ref }}=1\right)$. Simulation parameters in this case are: $\Delta t / t_{r e f}=0.1 \Delta x, n=16, p=4$, and $m=4$.

Figures $21 \mathrm{~d}$ and e show the reflected shock just after reflection and then shortly after it passes through the surface contact discontinuity. Note the contact discontinuity in the red circles at the extreme right of Fig. 21 e. The shock in this figure near $x=0$ is moving to the left.

The last four frames of Fig. 21 (f-i) show a shock, initially at $x=0.6$, moving to the right toward a contact discontinuity at $x=0.85$. In frame $(\mathrm{g})$, the oncoming shock has passed through the contact discontinuity at $x=0.9$ and has just contacted the right wall. A weak reflected shock off the contact surface is transmitting to the left at $x=0.75$. In frame (h), the strong shock has 


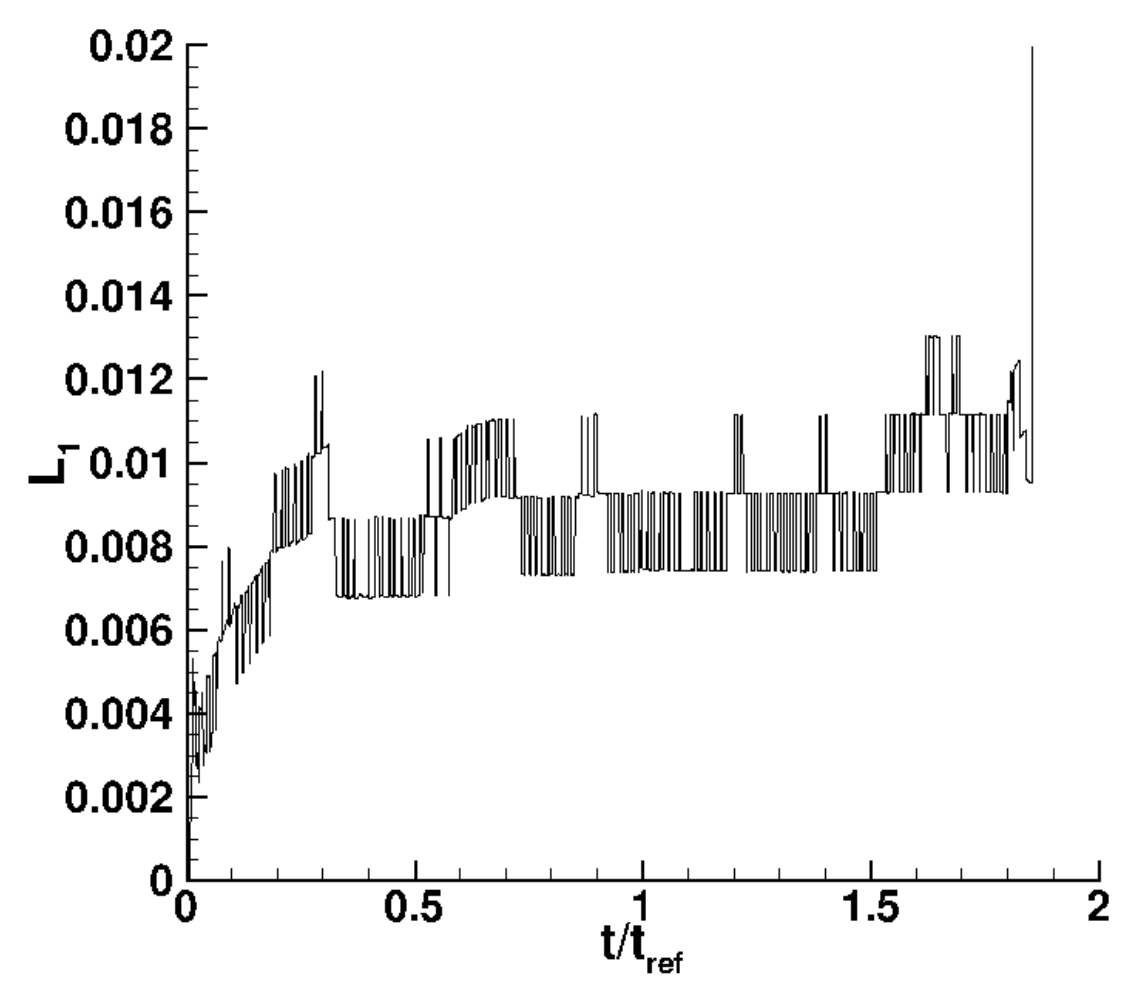

Figure 22. Error norm as a function of time for the shock tube problem, up to the first reflection.

reflected off the right wall and is overtaking the weak shock moving to the left near $x=0.35$. In frame (i), the strong shock has overtaken the weaker shock and a single wave continues movement to the left at $x=0$.

The purpose of this test is to evaluate how well the FWT algorithm tracks shocks and contact discontinuities in a highly unsteady environment that includes colliding features. The use of analytic relations to define and move discontinuities enables preservation of even weak jumps for long periods of time. It is observed that the target norm for accepting the FWT fit (Eq. 27) is not always achieved when multiple discontinuities occur in the same element. This condition is observed in the time shortly after the diaphragm is removed and in the reflection of a strong shock off the right wall and in the vicinity of the near stationary contact discontinuity. While the majority of elements are well resolved by the FWT, it appears that the algorithm for detecting two discontinuities in the same element requires more refinement.

In the current algorithm, conservation may be compromised every time a floater passes over a cell boundary. While the baseline formulation in smooth regions is conservative, there is currently no check that conservation is maintained when the floating boundary passes across a cell wall. While the exact Riemann solution is conservative, its implementation in discretized space requires additional constraints when the discontinuity passes over a cell center. These constraints are currently being investigated. A simple check of mass conservation in the current example should show that the integral of density with respect to distance from the contact discontinuity to the right wall should be a constant equal to 1.0. The integral of density over the entire domain, approximated by $\sum_{i=1}^{2^{p}} \rho_{i} \Delta x$, should be a constant equal to 7 . This integral over the entire domain varied between 
6.78 and 7.07 (-3.1\% to $1 \%$ error) over the time span of Fig. 21. (The same metric applied to the analytic solution up to the first reflection shows an error varying between $-0.7 \%$ and $0.3 \%$.) The integral of density from the contact surface to the right wall in Fig. 21(i) is 0.9675 (-3.25\% error).

\section{Concluding Remarks}

The principal contribution of this paper is the evaluation of a Fast Walsh Transform (FWT) curve fit to enable feature detection in the solution of nonlinear differential equations. The FWT fit is enabled through a new theorem, Walsh Root Function Annihilation of Low-Order Polynomials. That is, given a uniform distribution of $2^{p}$ cells on a one-dimensional element, it is proven that the inner product of the Walsh Root function for group $p$ with every polynomial of degree $\leq(p-1)$ across the element is identically zero. It is also proven that the magnitude and location of a discontinuous jump, as represented by a Heaviside function, are explicitly identified by its Fast Walsh Transform (FWT) coefficients. These two proofs enable an algorithm that quickly provides a Weighted Least Squares fit to distributions across the element that include a discontinuity. It is shown that flux reconstruction relative to the FWT fit in partial differential equations provides improved accuracy and eliminates the need for flux limiting in the vicinity of a discontinuity. The detection of a discontinuity further enables analytic relations to locally describe its evolution and provide increased accuracy.

Examples of inviscid flux formulation with respect to the FWT curve fits are provided for time-accurate advection, Burgers' equation, and quasi-one-dimensional Euler equations. An RK-4 method is used to advance all solutions in an explicit, time-accurate formulation. Fourth-order accuracy is confirmed for the advection of a Gaussian profile and for the steady-state solution of Burgers equation with a cell Reynolds number $\Delta x / \nu<1$. Discontinuities in a test profile translate across the domain twenty times without dissipation (jump across a single cell) and without any overshoot, maintaining second-order convergence. Discontinuities in the nonlinear sample problems are detected within a single cell, without ringing using the FWT fit formulation. The steady state limit for a standing shock in a de Laval nozzle is accurately recovered. The algorithm is able to detect and preserve shocks and surface contact discontinuities through multiple reflections and collisions within a closed, shock tube problem.

Some thirty years ago, the CFD community predominantly adopted upwind-based, shockcapturing methods over various implementations of shock-fitting methods, utilizing unsteady Rankine Hugoniot relations to compute analytic jump conditions and shock velocities. Shock-capturing algorithms make minor adjustments to a stencil based on local gradients to enable a stable discretization across the shock (limiting) but do not use any analytic information based on the Rankine Hugoniot equations. Shock-fitting algorithms must carry the infrastructure to define interior shock coordinates and make significant adjustments to the stencil at these interior boundaries, but they benefit from a more accurate, analytic representation of shocks moving through the domain. The vision for the FWT fit is to work like a shock-capturing method with respect to detection but work like a shock-fitting method with respect to use of more accurate analytic relations. The enabling innovation for feature detection is the ability to quantitatively define the magnitude and location of a discontinuity within some $2^{p}$ cells in an element within the domain using the FWT curve fit. The cost here is a larger stencil of cells required than for either shock capturing or shock fitting. The expectation is that the state of the art is moving toward use of higher-order elements so that the requisite stencil for detection is already available. 


\section{Acknowledgments}

This work was supported by the NASA Revolutionary Computational Aerosciences Program within the Transformational Tools and Technologies Project and by Game Changing Development Program under the Entry Systems Modeling Project .

\section{References}

\footnotetext{
${ }^{1}$ Candler, G.; Barnhardt, M.; Drayna, T.; Nompelis, I.; Peterson, D.; and Subbareddy, P.: Unstructured Grid Approaches for Accurate Aeroheating Simulations. AIAA Paper 2007-3959, June 2007.

${ }^{2}$ Barter, G. E.: Shock Capturing with PDE-Based Artificial Viscosity for an Adaptive, Higher-Order Discontinuous Galerkin Finite Element Method. MIT dissertation, May 2008.

${ }^{3}$ Gnoffo, P. A.: Updates to Multi-Dimensional Flux Reconstruction for Hypersonic Simulations on Tetrahedral Grids. AIAA Paper 2010-1271, Jan. 2010.

${ }^{4}$ Kontinos, D. A.; and McRae, D. S.: Rotated Upwind Strategies for Solution of the Euler Equations. AIAA Paper 94-0079, Jan. 1994.

${ }^{5}$ Dadone, A.; and Grossman, B.: Characteristic-Based, Rotated Upwind Scheme for the Euler Equations. AIAA J., Vol. 30, no. 9, 1992, pp. 2219-2226.

${ }^{6}$ Ren, Y.-X.: A robust shock-capturing scheme based on rotated Riemann solvers. Computers and Fluids, Vol. 32, 2003, pp. 1379-1403.

${ }^{7}$ Smits, A. J.; and Martin, M. P.: TURBULENCE IN SUPERSONIC AND HYPERSONIC BOUNDARY LAYERS. IUTAM Symposium on One Hundred Years of Boundary Layer Research: Proceedings of the IUTAM Symposium held at DLR-Göttingen, Germany, August 12-14, 2004.

${ }^{8}$ Gnoffo, P. A.; Thompson, K.; and Korzun, A.: Tapping the Brake for Entry, Descent, and Landing. AIAA Paper 2016-4277, Jun. 2016.

${ }^{9}$ F. D. Lora-Clavijo, J. P. Cruz-Perez, F. Siddhartha Guzman, and J. A. Gonzalez: Exact solution of the $1 \mathrm{D}$ riemann problem in Newtonian and relativistic hydrodynamics. Revista Mexicana de Fisica E, Vol. 59, 2013, pp. $28-50$.

${ }^{10}$ Walsh, J. L.: A Closed Set of Normal Orthogonal Functions. American Journal of Mathematics, Vol. 45, no. 1, Jan. 1923, pp. 5-24.

${ }^{11}$ Fine, N. J.: On the Walsh functions. Trans. Amer. Matc. Soc., Vol. 65, no. 3, May 1949, pp. 372-414.

${ }^{12}$ Adjouadi, M.; Sanchez, D.; Cabrerizo, M.; Ayala, M.; Jayakar, P.; Yaylali, I.; and Barreto, A.: Interictal Spike Detection Using the Walsh Transform. IEEE TRANSACTIONS ON BIOMEDICAL ENGINEERING, Vol. 51, no. 5, May 2004, pp. 868-872.

${ }^{13}$ Andrews, H. C.: Walsh Functions in Image Processing, Feature Selection and Pattern Recognition. IEEE TRANSACTIONS ON ELECTROMAGNETIC COMPATIBILITY, , no. 3, Aug. 1971, pp. $26-32$.

${ }^{14}$ Gnoffo, P. A.: Unsteady Solutions of NonLinear Differential Equations Using Walsh Function Series. AIAA Paper 2015-2756, NASA, June 2015.

${ }^{15}$ Gnoffo, P. A.: A Walsh Function Users' Manual. NASA TM 218536, NASA, Oct. 2014.

${ }^{16}$ Gnoffo, P. A.: Global Series Solutions of Nonlinear Differential Equations with Shocks Using Walsh Functions. J. Comput. Phys., Vol. 258, Feb 2014, pp. 650-688.

${ }^{17}$ Mazaheri, A.; and Nishikawa, H.: Efficient high-order discontinuous Galerkin schemes with first-order hyperbolic advectiondiffusion system approach. J. Comput. Phys., Vol. 321, Sept. 2016, pp. 729-754.

${ }^{18}$ Mazaheri, A. R.; Ricchiuto, M.; and Nishikawa, H.: Hyperbolic Method for Dispersive PDEs: Same High-Order of Accuracy for Solution, Gradient, and Hessian. AIAA Paper 2016-3970, June 2016.

${ }^{19}$ Gnoffo, P. A.: Solutions of NonLinear Differential Equations with Feature Detection Using Fast Walsh Transforms. J. Comput. Phys., Vol. 338, Jun 2017, pp. 620-649.

${ }^{20}$ Bonfiglioli, A.; Paciorri, R.; and Campoli, L.: Unsteady shock-fitting for unstructured grids. Int. J. Num. Meth. Fluids, Vol. 81, no. 4, June 2016, pp. 245-261.

${ }^{21}$ Pepe, R.; Bonfiglioli, A.; D'Angola, A.; Colonna, G.; and Paciorri, R.: An unstructured shock-fitting solver for hypersonic plasma flows in chemical non-equilibrium. Computer Physics Communications, Vol. 196, Nov. 2015, pp. $179-193$.

${ }^{22}$ Churchill, R. V.: Fourier Series and Boundary Value Problems. McGraw-Hill Book Company, 1969.

${ }^{23}$ Young, R. M.; and Gregory, R. T.: A Survey of Numerical Mathematics, Volume I. Addison-Wesley Publishing Company, 1972.
} 
${ }^{24}$ Carlson, J.-R.: Inflow/Outflow Boundary Conditions with Application to FUN3D. NASA TM 2011-217181, NASA, Oct. 2011.

${ }^{25}$ Ames Research Staff: Equations, Tables, and Charts for Compressible Flow. NACA TR 1135, 1953.

${ }^{26}$ Sod, G. A.: A Survey of Several Finite Difference Methods for Systems of Nonlinear Hyperbolic Conservation Laws. J. Comput. Phys., Vol. 27, 1978, pp. 1-31. 